\title{
TRACE DE CAUCHY POUR CERTAINES FONCTIONS LOCALEMENT INTÉGRABLES SUR UN OUVERT BORNÉ DE $\mathbb{C}$
}

\author{
IBAN HARLOUCHET
}

\begin{abstract}
Let $\Omega$ be a bounded open subset of $\mathbb{C}$, and let $f$ be a distribution on $\Omega$ such that $\bar{\partial} f$ is a Radon measure of finite total mass. By means of the Cauchy transform, we introduce the "Cauchy trace" of $f$, which takes values in the set of analytic functionals on the boundary $\partial \Omega$ of $\Omega$. The properties of this application are studied in detail. For instance, the characterization of its kernel is discussed according to the properties of the boundary $\partial \Omega$. Roughly speaking, the Cauchy trace allows us to interpret the CauchyPompeiu formula in the same way as the Sobolev trace allows to interpret the Stokes formula.
\end{abstract}

\section{Introduction}

Soit $\Omega$ un ouvert borné et régulier du plan complexe et soit $\Gamma=\partial \Omega$ la frontière de $\Omega$. Posons $\bar{\Omega}=\Omega \cup \Gamma$ et soit $f(z)$ une fonction de classe $\mathcal{C}^{1}$ sur $\bar{\Omega}$, par ailleurs arbitraire. Soit $F(z)$ la fonction qui est égale à $f(z)$ si $z \in \bar{\Omega}$ et à 0 si $z \notin \bar{\Omega}$. On a alors (les dérivées étant prises au sens des distributions)

$$
\bar{\partial} F=(\bar{\partial} f) \chi_{\Omega}+\frac{i}{2} f(z) d z_{\Gamma} .
$$

Puisque $\frac{1}{\pi z}$ est une solution fondamentale de $\bar{\partial}$, il vient

$$
f(z)=\frac{1}{\pi} \iint_{\Omega} \frac{\bar{\partial} f(\xi)}{z-\xi} d m(\xi)+\frac{1}{2 i \pi} \int_{\Gamma} \frac{f(\xi)}{\xi-z} d \xi
$$

2000 Mathematics Subject Classification. 30E25, 31, 46, 30A98.

Mots-clés. Transformée de Cauchy, fonctionnelle analytique, trace de Cauchy, intégrale de Poisson, mesure harmonique, approximation rationnelle, courbe rectifiable, capacité analytique. 
si $z \in \Omega$, tandis que

$$
0=\frac{1}{\pi} \iint_{\Omega} \frac{\bar{\partial} f(\xi)}{z-\xi} d m(\xi)+\frac{1}{2 i \pi} \int_{\Gamma} \frac{f(\xi)}{\xi-z} d \xi
$$

si $z \notin \bar{\Omega}$.

Les identités (2) et (3) sont les "célèbres identités de Cauchy". Le point de départ de notre travail consiste à interpréter (2) et (3) d'une façon un peu nouvelle.

Supposons que $f(z)$ soit une distribution appartenant à $\mathcal{D}^{\prime}(\Omega)$ telle que $\bar{\partial} f=\mu$ soit une mesure de Radon de masse totale finie. Nous nous proposons, dans ces conditions, de donner un sens à la trace de $f$ sur $\Gamma$ en définissant $\int_{\Gamma} f(z) R(z) d z$ pour toute fraction rationnelle $R(z)$ dont les pôles n'appartiennent pas à $\Gamma$.

On considère

$$
g(z)=f(z)-\frac{1}{\pi} \iint_{\Omega} \frac{d \mu(\xi)}{z-\xi},
$$

qui appartient à $\mathcal{D}^{\prime}(\Omega)$, et l'on a $\bar{\partial} g=0$, par construction. La distribution $g(z)$ est donc une fonction holomorphe dans $\Omega$. En particulier $g(z)$ est définie pour $z \in \Omega$. Il en est évidemment de même pour

$$
g(z)=-\frac{1}{\pi} \iint_{\Omega} \frac{d \mu(\xi)}{z-\xi} \quad(z \notin \bar{\Omega}) .
$$

Finalement les formules (2) et (3) permettent de donner un sens à

$$
\int_{\Gamma} \frac{f(z)}{\xi-z} d \xi \quad(z \notin \Gamma) .
$$

Plus généralement, soit $\Omega$ un ouvert borné quelconque de $\mathbb{C}$, soit $\mathcal{O}(\partial \Omega)$ l'algèbre des germes de fonctions holomorphes sur $\partial \Omega$, et soit $\mathcal{F}(\partial \Omega)$ l'espace de Fréchet des fonctions holomorphes sur $\mathbb{C} \backslash \partial \Omega$ s'annulant à l'infini. Le théorème de dualité de Silva [G, p. 47] permet d'identifier le dual de l'espace réflexif $\mathcal{O}(\partial \Omega)$ à $\mathcal{F}(\partial \Omega)$ (Théorème 2.1).

Soit $f \in \mathcal{D}^{\prime}(\Omega)$ telle que $\bar{\partial} f$ soit une mesure de Radon de masse totale finie. On définit la trace de $f$ sur $\partial \Omega$ comme étant l'élément $\mathcal{T}(f)$ de $\mathcal{F}(\partial \Omega)=\mathcal{O}^{\prime}(\partial \Omega)$ défini par la formule

$$
\left\{\begin{array}{l}
\mathcal{T} f_{\mid \Omega}=f-\left(\bar{\partial} f * \frac{1}{\pi z}\right)_{\mid \Omega} \\
\mathcal{T} f_{\mid \mathbb{C} \backslash \bar{\Omega}}=-\left(\bar{\partial} f * \frac{1}{\pi z}\right)_{|\mathbb{C}| \bar{\Omega}}
\end{array}\right.
$$


Posons $R_{z}(\xi)=\frac{1}{\xi-z}$ pour $\xi \neq z$. Dans ce cas, la distribution $f$ appartient à $\mathcal{L}_{\text {loc }}^{1}(\Omega)$ et on a

$$
f(z)=\frac{1}{\pi} \iint_{\Omega} \frac{d(\bar{\partial} f)(\xi)}{z-\xi}+\left\langle R_{z}, \mathcal{T}(f)\right\rangle
$$

pour presque tout $z \in \Omega$

$$
0=\frac{1}{\pi} \iint_{\Omega} \frac{d(\bar{\partial} f)(\xi)}{z-\xi}+\left\langle R_{z}, \mathcal{T}(f)\right\rangle
$$

si $z \notin \bar{\Omega}$

Les formules (2') et (3') sont des extensions très générales des formules (2) et (3) (il n'y a aucune hypothèse de régularité sur $\partial \Omega$ ), où $\frac{1}{2 i \pi} \int_{\Gamma} \frac{f(\xi)}{\xi-z} d \xi$ est remplacé $\operatorname{par}\left\langle R_{z}, \mathcal{T}(f)\right\rangle$.

En particulier, $2 i \pi\langle R, \mathcal{T}(f)\rangle$ "donne un sens" à $\int_{\partial \Omega} R(\xi) f(\xi) d \xi$ si $R$ est une fraction rationnelle à pôles hors de $\Omega$.

On appellera $\mathcal{T}(f)$ la trace de Cauchy de $f$ sur $\partial \Omega$. Cette notion de trace apparaît déjà dans $[\mathbf{E V 1}],[\mathbf{E V 2}]$ dans le cas où $\Omega$ est le disque unité $\mathbb{D}$. L'objet de cet article est d'étudier de manière systématique les propriétés de cette trace.

En un certain sens, $\mathcal{T}(f)$ dépend continûment de $\Omega$. Plus précisément si $\left(K_{n}\right)_{n \geq 1}$ est une suite exhaustive de compacts de $\Omega$ alors

$$
\langle g, \mathcal{T}(f)\rangle=\lim _{n \rightarrow \infty}\left\langle g, \mathcal{T}_{\partial K_{n}}\left(f_{\mid K_{n}}\right)\right\rangle
$$

uniformément sur tout borné de $\mathcal{O}(\partial \Omega)$ (Proposition 3.3).

D'autre part si on pose

$$
f^{*}=\mathcal{T}(f)_{\mid \Omega}+\left(\bar{\partial} f * \frac{1}{\pi z}\right)_{\mid \Omega},
$$

qui est égale presque partout à $f$ sur $\Omega$, il résulte du Lemme 3.4 que si $\partial K_{n}$ est de classe $\mathcal{C}^{1}$, et si

$$
\int_{\partial K_{n}}\left[\iint_{\Omega} \frac{|d \bar{\partial} f|(\xi)}{|z-\xi|}\right]|d z|<\infty
$$

alors

$$
\left\langle g, \mathcal{T}_{\partial K_{n}^{\circ}}\left(f_{\mid K_{n}}^{\circ}\right)\right\rangle=\frac{1}{2 i \pi} \int_{\partial K_{n}} f^{*}(\xi) g(\xi) d \xi \quad\left(g \in \mathcal{O}\left(\partial K_{n}\right)\right) .
$$

Dans certains cas on peut décrire de manière plus précise l'action de $\mathcal{T} f$ sur $g \in \mathcal{O}(\partial \Omega)$. Soit $\Omega$ un domaine simplement connexe de $\mathbb{C}$, 
et soit $\varphi: \mathbb{D} \rightarrow \Omega$ une représentation conforme de $\Omega$ sur le disque unité ouvert. Posons $\Omega_{r}=\varphi(r \mathbb{D})$. Alors

$$
\langle g, \mathcal{T} f\rangle=\lim _{r \rightarrow 1^{-}} \operatorname{ess} \frac{1}{2 i \pi} \int_{\partial \Omega_{r}} g(\xi) f(\xi) d \xi .
$$

En fait le Théorème 3.5 donne une formule analogue dans le cas où $\Omega$ est finiment connexe, basée sur l'équivalence conforme de $\Omega$ à un domaine circulaire.

Si de plus $f \in \mathcal{C}(\bar{\Omega})$ et si $\partial \Omega$ est une réunion finie disjointe de courbes de Jordan rectifiables, alors $\langle g, \mathcal{T}(f)\rangle=\frac{1}{2 i \pi} \int_{\partial \Omega} f(\xi) g(\xi) d \xi$ (Théorème 3.6) et on obtient notamment une version très générale des formules (2) et (3):

$$
\frac{1}{\pi} \iint_{\Omega} \frac{d(\bar{\partial} f)(\xi)}{z-\xi}+\frac{1}{2 i \pi} \int_{\partial \Omega} \frac{f(\xi)}{\xi-z} d \xi= \begin{cases}f(z) & \text { p.p. sur } \Omega \\ 0 & \text { sur } \mathbb{C} \backslash \bar{\Omega} .\end{cases}
$$

Soit $\mathcal{M}(\Omega)$ l'ensemble des mesures de Radon de masse totale finie sur $\Omega$ et soit $\mathcal{M}_{\text {loc }}(\Omega, \bar{\partial})=\left\{f \in \mathcal{L}_{\text {loc }}^{1}(\Omega): \bar{\partial} f \in \mathcal{M}(\Omega)\right\}$ le domaine de définition de la trace de Cauchy. On va maintenant décrire l'image de $\mathcal{M}_{\text {loc }}(\Omega, \bar{\partial})$ par la trace de Cauchy. Ceci revient à caractériser

$$
\mathcal{C}^{-}(\mathcal{M}(\Omega))=\left\{\left(\mu * \frac{1}{\pi z}\right)_{\mid \mathbb{C} \backslash \bar{\Omega}}\right\}_{\mu \in \mathcal{M}(\Omega)} .
$$

Dans le cas du disque unité $\mathbb{D}$, il est connu que $\mathcal{C}^{-}(\mathcal{M}(\mathbb{D}))=\mathcal{P}^{-}\left(\mathcal{L}^{1}(\mathbb{T})\right)$, où

$$
\mathcal{P}^{-}(h)(z)=\frac{1}{2 i \pi} \int_{\mathbb{T}} \frac{h(\xi)}{\xi-z} d \xi
$$

pour $h \in \mathcal{L}^{1}(\mathbb{T}),|z|>1[\mathbf{N}],[\mathbf{S}]$.

La clef de la description de $\mathcal{C}^{-}(\mathcal{M}(\Omega))$ pour un ouvert borné quelconque de $\mathbb{C}$ est donnée par une propriété connue, mais difficile à trouver dans la littérature, des mesures harmoniques.

Si $U$ est un domaine borné de $\mathbb{C}$ et si $h \in \mathcal{C}(\partial U)$, la fonction de Perron $H$ de $h$ vérifie pour $z \in U$ la formule

$$
H(z)=\int_{\partial U} h(\xi) d \omega_{z}(\xi),
$$

où $\omega_{z}$ est la mesure harmonique en $z$ relativement à $U$. Soit $\omega=\omega_{z_{0}}$, avec $z_{0} \in U$. On déduit de [Ra] que l'application $h \rightarrow \int_{\partial U} h(\xi) d \omega_{z}(\xi)$ est une isométrie de $\mathcal{L}^{\infty}(\partial U, \omega)$ dans l'espace des fonctions harmoniques bornées sur $U$. 
Soit maintenant $\Omega$ un ouvert borné quelconque de $\mathbb{C}$, et soit $\left(\Omega_{n}\right)_{0 \leq n<p \leq \infty}$ la famille finie ou dénombrable des composantes connexes de $\Omega$. Pour $0 \leq n<p$, choisissons arbitrairement $z_{n} \in \Omega_{n}$, et soit $\omega_{z_{n}}$ la mesure harmonique de $\Omega_{n}$ en $z_{n}$, étendue à $\partial \Omega$ par la formule $\omega_{z_{n}}\left(\partial \Omega \backslash \partial \Omega_{n}\right)=0$. On associe à $\Omega$ la mesure borélienne

$$
\mu=\sum_{0 \leq n<p} \frac{\omega_{z_{n}}}{2^{n}}
$$

et pour $f \in \mathcal{L}^{1}(\partial \Omega, \mu)$ on pose

$$
\mathcal{P}^{-}(f)(z)=\frac{1}{2 i \pi} \int_{\partial \Omega} \frac{f(\xi)}{\xi-z} d \mu(\xi) \quad(z \notin \bar{\Omega}) .
$$

Alors $\mathcal{C}^{-}(\mathcal{M}(\Omega))=\mathcal{P}^{-}\left(\mathcal{L}^{1}(\partial \Omega, \mu)\right)$ et l'image de l'application trace est égale à $\mathcal{H}(\Omega) \oplus \mathcal{P}^{-}\left(\mathcal{L}^{1}(\partial \Omega, \mu)\right)$.

Dans le cas où $\Omega$ est un domaine borné dont la frontière est une réunion finie disjointe de courbes de Jordan rectifiables, le résultat précédent est valable pour $\mu=|d z|$, car les mesures harmoniques $\omega_{z}$ et la mesure longueur d'arc $|d z|$ sont alors mutuellement absolument continues. Notons que si de plus les courbes de Jordan sont régulières au sens d'Ahlfors, alors $\mathcal{P}^{-}(f)$ admet des limites non tangentielles presque partout sur $\partial \Omega$ pour $f \in \mathcal{L}^{1}(\partial \Omega,|d z|)$, et $\mathcal{P}^{-}(f)(z)$ est la " $A$-intégrale" de Cauchy de sa limite radiale pour $z \notin \bar{\Omega}$ (Remarque 4.6(2)). Ceci permet, dans ce cas, d'interpréter $\mathcal{C}^{-}(\nu), \nu \in \mathcal{M}(\Omega)$, comme la fonction $g \in \mathcal{L}_{0}^{1, \infty}(\partial \Omega)$ définie presque partout sur $\partial \Omega$ par la formule $g(\xi)=$ $\lim$ n.t. $z \rightarrow \xi \mathcal{C}^{-}(\nu)(z)$.

On note $\mathcal{M}_{c}(\Omega, \bar{\partial})$ l'ensemble des fonctions de $\mathcal{M}_{\text {loc }}(\Omega, \bar{\partial})$ à support compact. On a alors

$$
\mathcal{M}_{c}(\Omega, \bar{\partial}) \subset \operatorname{Ker} \mathcal{T} \subset \mathcal{L}^{1}(\Omega) .
$$

Les séries de Wolff-Denjoy du disque donnent des exemples non triviaux de fonctions de $\mathcal{M}_{\text {loc }}(\mathbb{D}, \bar{\partial})$ dont la trace de Cauchy est nulle. Soit $v_{c}$ (resp. $v$, resp. $v_{\infty}$ ) la topologie de la convergence simple sur $C_{c}(\Omega)$ (resp. $\mathcal{C}(\bar{\Omega})$, resp. $\mathcal{C}_{b}(\Omega)$ ). Notons $\|\cdot\|_{1}$ la norme $\mathcal{L}^{1},\|\cdot\|$ la norme sur $\mathcal{M}(\Omega)$, et soit $w$ la topologie duale faible de $\mathcal{F}(\partial \Omega)$. L'application $\mathcal{T}$ étant $\left(v_{c}, v\right)$-w-continue (Proposition 5.1), on a

$$
{\overline{\mathcal{M}_{c}(\Omega, \bar{\partial})}}^{\left(\|\cdot\|_{1},\|\cdot\|\right)} \subset{\overline{\mathcal{M}_{c}(\Omega, \bar{\partial})}}^{\left(v_{c}, v_{\infty}\right)} \subset{\overline{\mathcal{M}_{c}(\Omega, \bar{\partial})}}^{\left(v_{c}, v\right)} \subset \operatorname{Ker} \mathcal{T} .
$$


Le but poursuivi dans l'étude du noyau de $\mathcal{T}$ (Section 5) est de chercher sous quelles conditions sur $\Omega$ les inclusions ci-dessus sont des égalités.

Notons $\mathcal{A}(\Omega)$ l'algèbre des fonctions analytiques sur $\Omega$ continues sur $\bar{\Omega}$, $\mathcal{R}(\bar{\Omega})$ l'adhérence dans $\mathcal{A}(\Omega)$ des fonctions rationnelles à pôles hors de $\bar{\Omega}$ et $\mathcal{H}^{\infty}(\Omega)$ l'algèbre des fonctions analytiques bornées sur $\Omega$. On vérifie que pour que $\mathcal{M}_{c}(\Omega, \bar{\partial})$ soit $\left(v_{c}, v\right)$ - (resp. $\left(v_{c}, v_{\infty}\right)$-) dense dans $\operatorname{Ker} \mathcal{T}$, il faut que $\mathcal{R}(\bar{\Omega})$ soit $w^{*}$-dense dans $\mathcal{A}(\Omega)$ (resp. $\mathcal{H}^{\infty}(\Omega)$ ). Ceci renvoit à des questions classiques de la théorie des algèbres uniformes et de l'approximation rationnelle, liées à la théorie de la capacité analytique. En utilisant une construction de [Ga, p. 220], on peut en particulier construire un ouvert connexe $\Omega=\stackrel{\circ}{\Omega}$ tel que $\mathcal{M}_{c}(\Omega, \bar{\partial})$ ne soit pas $\left(v_{c}, v\right)$-dense dans $\operatorname{Ker} \mathcal{T}$ (Proposition 5.3).

Dans la direction opposée, on montre au Théorème 5.5 que si $\Omega$ est un domaine de Jordan borné, alors $\mathcal{M}_{c}(\Omega, \bar{\partial})$ est $\left(\|\cdot\|_{1},\|\cdot\|\right)$-dense dans $\operatorname{Ker} \mathcal{T}$. Ce résultat équivaut au suivant: toute mesure $\nu \in \mathcal{M}(\Omega)$ telle que $\mathcal{C}^{-}(\nu) \equiv 0$ est limite en norme d'une suite $\left(\mu_{n}\right)$ de mesures à support compact dans $\Omega$ telle que $\mathcal{C}^{-}\left(\mu_{n}\right) \equiv 0$. Pour $0<r<1$ définissons $\Omega_{r}$ comme plus haut. La mesure $\nu_{r}=\nu_{\mid \Omega_{r}}$ est à support compact dans $\Omega$, et $\lim _{r \rightarrow 1^{-}}\left\|\nu_{r}-\nu\right\|=0$. Le problème est que $\mathcal{C}^{-}\left(\nu_{r}\right)$ est en général non nulle. La démonstration repose sur le fait qu'on peut approcher convenablement $\nu_{r}$ par une mesure $\mu_{r} \in \mathcal{M}_{c}(\Omega)$ telle que $\mathcal{C}^{-}\left(\mu_{r}\right) \equiv 0$. Ce procédé d'approximation, assez technique, repose sur la théorie des espaces de Hardy. Ce théorème est également valable pour les domaines bornés dont le bord est une réunion finie disjointe de courbes de Jordan.

Du Théorème 5.5 on déduit en particulier que $\mathcal{D}(\Omega)$ est $\left(v_{c}, v\right)$-dense dans $\operatorname{Ker} \mathcal{T}$ (Corollaire 5.7). Comme le sous-espace $\mathcal{C}^{\infty}(\bar{\Omega})$ est $\left(v_{c}, v\right)$ dense dans $\mathcal{M}_{\text {loc }}(\Omega, \bar{\partial})$ (Remarque 5.8), la trace de Cauchy n'est autre que l'extension par continuité à $\left(\mathcal{M}_{\text {loc }}(\Omega, \bar{\partial}),\left(v_{c}, v\right)\right)$ de l'application $f \rightarrow$ $f_{\mid \partial \Omega} \operatorname{sur} \mathcal{C}^{\infty}(\bar{\Omega})$. Cette trace est donc une trace au sens le plus classique du terme [LM, Chapitre 1] dans le cas où $\Omega$ est un domaine de Jordan.

Cet article est issu d'un développement du Chapitre II de la thèse de l'auteur $[\mathbf{H}]$ préparée sous la direction de J. Esterle, et donnant des résultats partiels dans le cas du disque. Des remarques pertinentes de Y. Meyer sur la première version de cet article ont amené l'auteur à réorganiser complètement cet article et à s'intéresser aux ouverts bornés généraux. L'auteur tient à remercier Y. Meyer pour ses remarques et J. Esterle pour l'aide qu'il lui a apportée pendant toute la préparation de ce travail. 


\section{Fonctionnelles analytiques et transformée de Cauchy}

\subsection{Fonctionnelles analytiques.}

Soit $U$ un ouvert de $\mathbb{C}$; on note $\mathcal{H}(U)$ l'algèbre des fonctions holomorphes sur $U$. Si $\mathbb{C} \backslash U$ est borné, on notera $\mathcal{H}_{0}(U)$ l'algèbre des fonctions holomorphes sur $U$ nulles à l'infini.

Soit $\Omega$ un ouvert borné de $\mathbb{C}$; on note $\mathcal{O}(\partial \Omega)$ l'algèbre des germes de fonctions analytiques sur $\partial \Omega$, que l'on identifie à l'algèbre topologique $\varliminf_{U \supset \partial \Omega} \mathcal{H}(U)$ où $U$ parcourt l'ensemble des voisinages ouverts de $\partial \Omega$.

On notera $\mathcal{F}(\partial \Omega)$ l'espace $\mathcal{H}_{0}(\mathbb{C} \backslash \partial \Omega)$ muni de la topologie de la convergence uniforme sur tout compact, qui est un espace de Fréchet. Dans le cas où $\partial \Omega$ est analytique réelle, $\mathcal{F}(\Omega)$ est l'espace usuel des hyperfonctions sur $\partial \Omega$ (cf. [BG2]).

Soient $\varphi \in \mathcal{F}(\Omega), g \in \mathcal{O}(\partial \Omega)$ et $V$ un voisinage ouvert de $\partial \Omega$ tel que $\gamma:=\partial V$ soit une réunion finie disjointe de courbes de Jordan rectifiables (cf. par exemple [Tr]) et tel que $g$ soit analytique sur un voisinage de $\bar{V}$. On munit $\gamma$ du sens de parcours positif autour de $\bar{V}$, et on pose

$$
\langle g, \varphi\rangle=-\frac{1}{2 i \pi} \int_{\gamma} g(\xi) \varphi(\xi) d \xi .
$$

L'intégrale ci-dessus ne dépend pas du choix de $V$, et on a (cf. $[\mathbf{G}$, p. 47]):

Théorème 2.1 (J. Silva). Par le crochet de dualité défini ci-dessus, le dual de l'espace $\mathcal{O}(\partial \Omega)$ (resp. $\mathcal{F}(\partial \Omega)$ ) s'identifie à l'espace $\mathcal{F}(\partial \Omega)$ (resp. $\mathcal{O}(\partial \Omega)$ ).

Pour $z \in \mathbb{C} \backslash \partial \Omega, \xi \in \mathbb{C} \backslash\{z\}$, on pose $R_{z}(\xi)=\frac{1}{\xi-z}$, de sorte que $R_{z} \in \mathcal{O}(\partial \Omega)$.

Soient $\varphi \in \mathcal{F}(\partial \Omega), z \in \mathbb{C} \backslash \partial \Omega$, et soient $\rho>0$ tel que $\bar{B}(z, \rho) \cap \partial \Omega=\emptyset$, $R>\max \left(\sup _{\xi \in \partial \Omega}|\xi|,|z|+\rho\right), V=B(0, R) \backslash \bar{B}(z, \rho)$ et $\gamma=\partial V$. D'après la formule de Cauchy, on a alors

$$
\left\langle R_{z}, \varphi\right\rangle=-\frac{1}{2 i \pi} \int_{C(0, R)} \frac{\varphi(\xi)}{\xi-z} d \xi+\frac{1}{2 i \pi} \int_{C(z, \rho)} \frac{\varphi(\xi)}{\xi-z} d \xi=\varphi(z) .
$$

Donc

$$
\left\langle R_{z}, \varphi\right\rangle=\varphi(z) \quad(\varphi \in \mathcal{F}(\partial \Omega), z \in \mathbb{C} \backslash \partial \Omega) .
$$

En particulier,

$$
\operatorname{Span}\left\{R_{z}: z \in \mathbb{C} \backslash \partial \Omega\right\}=\mathcal{O}(\partial \Omega),
$$

Span désignant ici l'enveloppe linéaire fermée. 
On donne ci-dessous deux exemples de fonctionnelles analytiques:

(1) Supposons que $\partial \Omega$ soit une réunion finie disjointe de courbes de Jordan rectifiables. Soit $f \in \mathcal{L}^{1}(\partial \Omega)$ et soit

$$
\begin{aligned}
\varphi(f): \mathcal{O}(\partial \Omega) & \longrightarrow \mathbb{C} \\
g & \longrightarrow \frac{1}{2 i \pi} \int_{\partial \Omega} g(\xi) f(\xi) d \xi,
\end{aligned}
$$

alors $\varphi(f) \in \mathcal{O}^{\prime}(\partial \Omega)$, et l'application $\varphi: \mathcal{L}^{1}(\partial \Omega) \hookrightarrow \mathcal{O}^{\prime}(\partial \Omega)$ est continue. Comme $\mathcal{O}(\partial \Omega)$ est dense dans $\mathcal{C}(\partial \Omega), \varphi$ est injective.

(2) Supposons de plus que $\partial \Omega$ soit de classe $\mathcal{C}^{\infty}$ et considérons $\mathcal{D}(\partial \Omega)=$ $\mathcal{C}^{\infty}(\partial \Omega)$. Comme $\mathcal{O}(\partial \Omega)$ est dense dans $\mathcal{D}(\partial \Omega)$, l'espace des distributions $\mathcal{D}^{\prime}(\partial \Omega)$ s'injecte continûment dans $\mathcal{O}^{\prime}(\partial \Omega)$. Les fonctionnelles analytiques généralisent donc les notions de fonction intégrable et de distribution.

Posons $\mathcal{O}^{+}(\partial \Omega)=\underline{\lim }_{U \supset \bar{\Omega}} \mathcal{H}(U)$ où $U$ parcourt l'ensemble des voisinages ouverts de $\bar{\Omega}$, et $\mathcal{O}^{-}(\partial \Omega)=\underline{\lim } \mathcal{H}_{0}(\mathbb{C} \backslash K)$ où $K$ parcourt l'ensemble des parties compactes de $\Omega$. Soit $\vec{g} \in \mathcal{O}(\partial \Omega)$; avec les mêmes notations que dans (2.1), posons $\gamma^{+}=\gamma \cap \Omega, \gamma^{-}=\gamma \cap \complement \bar{\Omega}$, munis de l'orientation induite par celle de $\gamma$, et

$$
\begin{array}{ll}
g^{+}(z)=-\frac{1}{2 i \pi} \int_{\gamma^{-}} \frac{g(\xi)}{\xi-z} d \xi & (z \in \Omega \cup V), \\
g^{-}(z)=-\frac{1}{2 i \pi} \int_{\gamma^{+}} \frac{g(\xi)}{\xi-z} d \xi & (z \in \complement \bar{\Omega} \cup V) .
\end{array}
$$

Alors $g^{+} \in \mathcal{O}^{+}(\partial \Omega), g^{-} \in \mathcal{O}^{-}(\partial \Omega)$ et $g^{+}(z)+g^{-}(z)=g(z)$, pour $z \in V$, donc

$$
g=g^{+}+g^{-} .
$$

D'après le théorème de Liouville, la décomposition ci-dessus est unique. Notons qu'on a en particulier

$$
\begin{aligned}
& R_{z}=R_{z}^{+} \quad(z \in \mathbb{C} \backslash \bar{\Omega}), \\
& R_{z}=R_{z}^{-} \quad(z \in \Omega) \text {. }
\end{aligned}
$$

Soit $\mathcal{F}^{+}(\partial \Omega)=\left\{\varphi \in \mathcal{F}(\partial \Omega): \varphi_{\mid \mathbb{C} \backslash \bar{\Omega}}=0\right\}$ et $\mathcal{F}^{-}(\partial \Omega)=\{\varphi \in \mathcal{F}(\partial \Omega)$ : $\left.\varphi_{\mid \Omega}=0\right\}$. On a trivialement

$$
\mathcal{F}(\partial \Omega)=\mathcal{F}^{+}(\partial \Omega) \oplus \mathcal{F}^{-}(\partial \Omega)
$$


Pour $\varphi \in \mathcal{F}(\partial \Omega)$, notons $\varphi^{+}$la fonction holomorphe égale à $\varphi$ sur $\Omega$, nulle sur $\mathbb{C} \backslash \bar{\Omega}$. De même, notons $\varphi^{-}$la fonction holomorphe égale à $\varphi$ sur $\mathbb{C} \backslash \bar{\Omega}$, nulle sur $\Omega$, de sorte que $\varphi=\varphi^{+}+\varphi^{-}$. On a alors

$$
\left\langle g^{+}, \varphi^{+}\right\rangle=0, \quad\left\langle g^{-}, \varphi^{-}\right\rangle=0 \quad(g \in \mathcal{O}(\partial \Omega), \varphi \in \mathcal{F}(\partial \Omega)) .
$$

Le crochet de dualité (2.1) devient

$$
\langle g, \varphi\rangle=-\frac{1}{2 i \pi} \int_{\gamma^{+}} g^{-}(\xi) \varphi^{+}(\xi) d \xi-\frac{1}{2 i \pi} \int_{\gamma^{-}} g^{+}(\xi) \varphi^{-}(\xi) d \xi .
$$

Et on a les relations d'orthogonalité suivantes

$$
\mathcal{F}^{ \pm}(\partial \Omega)^{\perp}=\mathcal{O}^{\mp}(\partial \Omega), \quad \mathcal{O}^{ \pm}(\partial \Omega)^{\perp}=\mathcal{F}^{\mp}(\partial \Omega) .
$$

\subsection{Transformée de Cauchy de mesures.}

Soit $U$ un ouvert de $\mathbb{C}$ et soit $\mathcal{C}_{0}(U)$ l'espace des fonctions continues sur $U$, nulles à l'infini. On note $\mathcal{M}(U)$ l'espace des mesures complexes sur $U$. Muni de la norme $\|\nu\|=|\nu|(U)$, où $|\nu|$ est la variation totale de $\nu, \mathcal{M}(U)$ est un espace de Banach. D'après le théorème de Riesz, on peut identifier $\mathcal{M}(U)$ au dual de $\mathcal{C}_{0}(U)$. D'après le théorème de Radon-Nikodym, l'espace $\mathcal{L}^{1}(U)$ s'identifie isométriquement au sousespace fermé de $\mathcal{M}(U)$ formé des mesures absolument continues par rapport à la mesure de Lebesgue.

Notons $\mathcal{M}_{c}(\mathbb{C})$ l'espace des mesures complexes sur $\mathbb{C}$ à support compact muni de la topologie naturelle limite inductive des topologies des $\mathcal{M}_{K}(\mathbb{C})$ (espaces des mesures à support contenu dans $K$ ), où $K$ parcourt l'ensemble des compacts de $\mathbb{C}$. Soient $\nu \in \mathcal{M}(\mathbb{C})$ et $f \in \mathcal{L}^{1}(\mathbb{C})$; d'après le théorème de Fubini, l'intégrale $(\nu * f)(\xi)=\iint_{\mathbb{C}} f(\xi-\eta) d \nu(\eta)$ est définie presque partout sur $\mathbb{C}$ et l'application $\nu \rightarrow \nu * f, \mathcal{M}(\mathbb{C}) \rightarrow \mathcal{L}^{1}(\mathbb{C})$ est continue. De même, si $\nu \in \mathcal{M}_{c}(\mathbb{C})$ et $f \in \mathcal{L}_{\text {loc }}^{1}(\mathbb{C})$, l'intégrale $(\nu * f)(\xi)=$ $\iint_{\mathbb{C}} f(\xi-\eta) d \nu(\eta)$ est définie presque partout sur $\overline{\operatorname{Supp} \nu}$, partout ailleurs dans $\mathbb{C}$, et l'application $\nu \rightarrow \nu * f, \mathcal{M}_{c}(\mathbb{C}) \rightarrow \mathcal{L}_{\text {loc }}^{1}(\mathbb{C})$ est continue.

Soit $\beta \in \mathcal{L}_{\text {loc }}^{1}(\mathbb{C})$ la fonction $\xi \rightarrow \frac{1}{\pi \xi}$. On appelle transformée de Cauchy de $\nu \in \mathcal{M}_{c}(\mathbb{C})$ la fonction définie presque partout par la formule

$$
\mathcal{C}(\nu)(z)=(\nu * \beta)(z)=\frac{1}{\pi} \iint_{\mathbb{C}} \frac{d \nu(\xi)}{z-\xi}
$$

$\mathcal{C}(\nu)$ est définie presque partout sur $\overline{\operatorname{Supp} \nu}, \mathcal{C}(\nu) \in \mathcal{H}_{0}(\mathbb{C} \backslash \overline{\operatorname{Supp} \nu})$ et l'application $\mathcal{C}: \mathcal{M}_{c}(\mathbb{C}) \rightarrow \mathcal{L}_{\text {loc }}^{1}(\mathbb{C})$ est continue.

Soit maintenant $\Omega$ un ouvert borné de $\mathbb{C}$ et soit $\nu \in \mathcal{M}(\Omega)$. On note $\tilde{\nu}$ la prolongée de $\nu$ à $\mathbb{C}$ par 0 (i.e. $\tilde{\nu}(B)=\nu(B \cap \Omega)$ pour $B$ borélien de $\mathbb{C}$ ). 
La transformée de Cauchy de $\nu$ est alors définie par la formule

$$
\mathcal{C}(\nu)=\mathcal{C}(\tilde{\nu}) .
$$

Dans tout l'article, on notera

$$
\mathcal{C}^{+}(\nu)=\mathcal{C}(\nu)_{\mid \Omega}, \quad \mathcal{C}^{-}(\nu)=\mathcal{C}(\nu)_{|\mathbb{C}| \bar{\Omega}} .
$$

D'après la formule de Cauchy-Pompeiu, l'application $\beta$ est une solution fondamentale de $\bar{\partial}$, donc $\bar{\partial} \mathcal{C}(\nu)=\tilde{\nu}$. Inversement, si $f \in \mathcal{L}_{\text {loc }}^{1}(\mathbb{C})$ est à support compact, et si $\bar{\partial} f \in \mathcal{M}_{c}(\mathbb{C})$, alors $\bar{\partial}(f-\mathcal{C}(\bar{\partial} f)) \equiv 0$, donc $f-\mathcal{C}(\bar{\partial} f)$ est entière (cf. [BG1, p. 252]). Comme $f$ et $\mathcal{C}(\bar{\partial} f)$ s'annulent à l'infini, on a $\mathcal{C}(\bar{\partial} f)=f$.

Pour $\nu \in \mathcal{M}(\Omega)$, on a vu que $\mathcal{C}(\nu)$ est holomorphe sur $\mathbb{C} \backslash \overline{\operatorname{Supp} \nu}$. On a aussi des propriétés de régularité sur $\overline{\operatorname{Supp} \nu}$. Si $u \in \cup_{q>2} \mathcal{L}^{q}(\Omega)$, il est clair que $\mathcal{C}(u) \in \mathcal{C}(\mathbb{C})$, et si $u \in \mathcal{C}^{k}(\Omega)$ alors $\mathcal{C}^{+}(u) \in \mathcal{C}^{k}(\Omega)(0 \leq k \leq \infty)$ (cf. [BG1, p. 99]).

\section{Trace de Cauchy}

Dans tout ce qui suit, le $\bar{\partial}$ est calculé au sens des distributions. Notons que si $f \in \mathcal{D}^{\prime}(\Omega)$ et si $\bar{\partial} f \in \mathcal{M}(\Omega)$ alors $f-\mathcal{C}(\bar{\partial} f)$ coïncide avec une fonction holomorphe sur $\Omega$. On identifie alors la distribution $f-\mathcal{C}(\bar{\partial} f)$ avec cette fonction holomorphe, et $f$ est la distribution associée à $\left[f-\mathcal{C}^{+}(\bar{\partial} f)\right]+\mathcal{C}^{+}(\bar{\partial} f) \in \mathcal{L}_{\text {loc }}^{1}(\Omega)$.

Définition 3.1. Soit $\Omega$ un ouvert borné de $\mathbb{C}$ et soit

$$
\mathcal{M}_{\mathrm{loc}}(\Omega, \bar{\partial})=\left\{f \in \mathcal{L}_{\mathrm{loc}}^{1}(\Omega): \bar{\partial} f \in \mathcal{M}(\Omega)\right\} .
$$

On appelle trace de Cauchy sur $\Omega$ l'application

$$
\begin{aligned}
\mathcal{T}: \mathcal{M}_{\mathrm{loc}}(\Omega, \bar{\partial}) & \longrightarrow \mathcal{F}(\partial \Omega) \\
f & \longrightarrow \mathcal{T}(f)
\end{aligned}
$$

définie par $\mathcal{T}(f)^{+}=f-\mathcal{C}^{+}(\bar{\partial} f)$ et $\mathcal{T}(f)^{-}=-\mathcal{C}^{-}(\bar{\partial} f)$.

Remarques 3.2.

(1) $\mathcal{M}_{\text {loc }}(\Omega, \bar{\partial})$ muni de la topologie induite par $\mathcal{L}_{\text {loc }}^{1}(\Omega)$ et $\mathcal{M}(\Omega)$ est un espace de Fréchet, et l'application linéaire $\mathcal{T}: \mathcal{M}_{\text {loc }}(\Omega, \bar{\partial}) \rightarrow \mathcal{F}(\partial \Omega)$ est continue, car sur $\mathcal{H}(\Omega)$ la topologie de la convergence uniforme sur tout compact et la topologie $\mathcal{L}_{\text {loc }}^{1}(\Omega)$ coïncident.

(2) Si $f \in \mathcal{C}^{1}(\bar{\Omega})$ et si $\partial \Omega$ est de classe $\mathcal{C}^{1}$ par morceaux (de sorte que $\partial \Omega$ est une réunion finie disjointe de courbes de Jordan de classe $\mathcal{C}^{1}$ 
par morceaux), alors d'après la formule de Cauchy-Pompeiu

$$
\mathcal{T}(f)(z)=\frac{1}{2 i \pi} \int_{\partial \Omega} \frac{f(\xi)}{\xi-z} d \xi \quad(z \in \mathbb{C} \backslash \partial \Omega) .
$$

Or, d'après (2.2),

$$
\left\langle R_{z}, \mathcal{T} f\right\rangle=\mathcal{T} f(z) \quad(z \in \mathbb{C} \backslash \partial \Omega) .
$$

Donc $\langle g, \mathcal{T} f\rangle=\frac{1}{2 i \pi} \int_{\partial \Omega} f(\xi) g(\xi) d \xi$ pour $g \in \mathcal{O}(\partial \Omega)$, et $\mathcal{T}(f)=f_{\mid \partial \Omega}$.

(3) Notons limn. $z \rightarrow z_{0}$ la limite en $z_{0}$ le long de la direction normale à $\Gamma$. Si $\Omega$ est un domaine de Jordan $\mathcal{C}^{1}$-régulier, et si $f \in \mathcal{C}^{1}(\bar{\Omega})$, alors, d'après (3.1) et la formule de Plemelj-Sokhotski [BG1, p. 267], on a

$$
\operatorname{limn}_{z \rightarrow z_{0}, z \in \Omega} \mathcal{T}(f)^{+}(z)-\operatorname{limn}_{z \rightarrow z_{0}, z \notin \bar{\Omega}} \mathcal{T}(f)^{-}(z)=f\left(z_{0}\right) \quad\left(z_{0} \in \partial \Omega\right) .
$$

Dans la suite, on va notamment montrer que l'égalite $\mathcal{T}(f)=f_{\mid \partial \Omega}$ est vraie dans un cadre beaucoup plus général.

Proposition 3.3. Soient $\Omega$ un ouvert borné de $\mathbb{C}, f \in \mathcal{M}_{\mathrm{loc}}(\Omega, \bar{\partial})$ et $\left(K_{n}\right)_{n \in \mathbb{N}}$ une suite exhaustive de compacts de $\Omega$. Alors

$$
\langle g, \mathcal{T}(f)\rangle=\lim _{n \rightarrow \infty}\left\langle g, \mathcal{T}_{\partial \check{K}_{n}}\left(f_{\mid \stackrel{\circ}{K_{n}}}\right)\right\rangle
$$

uniformément sur tout borné de $\mathcal{O}(\partial \Omega)$.

Démonstration: Posons $\partial \stackrel{\circ}{K_{n}}=\gamma_{n}$ et $f_{\mid \stackrel{\circ}{K_{n}}}=f_{n}$. Soient $z \in \mathbb{C} \backslash \partial \Omega$ et $R>0$ tels que $\bar{B}(z, R) \subset \mathbb{C} \backslash \bar{\Omega}$ ou tels que $\bar{B}(z, R) \subset \Omega$. Dans le deuxième cas, $\bar{B}(z, R) \subset \stackrel{\circ}{K}_{n}$ pour $n$ assez grand. On va montrer que $\mathcal{T}_{\gamma_{n}}\left(f_{n}\right)$ converge uniformément vers $\mathcal{T}(f)$ sur $\left.\bar{B}(z, r), r \in\right] 0, R$. Soit $\rho \in] r, R[$; d'après la formule intégrale de Cauchy, pour $\xi \in \bar{B}(z, r)$,

$$
\begin{aligned}
& \mathcal{T}(f)(\xi)-\mathcal{T}_{\gamma_{n}}\left(f_{n}\right)(\xi) \\
& \quad=\frac{1}{2 i \pi} \int_{0}^{2 \pi} \frac{\mathcal{T}(f)\left(z+\rho e^{i t}\right)-\mathcal{T}_{\gamma_{n}}\left(f_{n}\right)\left(z+\rho e^{i t}\right)}{z+\rho e^{i t}-\xi} i \rho e^{i t} d t .
\end{aligned}
$$

En moyennisant l'expression de droite sur $] \rho_{1}, R\left[, \rho_{1}>r\right.$, et en posant $A\left(z, \rho_{1}, R\right)=\left\{u \in \mathbb{C}: \rho_{1}<|u-z|<R\right\}$, il vient

$$
\begin{aligned}
& \mathcal{T}(f)(\xi)-\mathcal{T}_{\gamma_{n}}\left(f_{n}\right)(\xi) \\
& \quad=\frac{1}{2 \pi\left(R-\rho_{1}\right)} \iint_{A\left(z, \rho_{1}, R\right)} \frac{\mathcal{T}(f)(u)-\mathcal{T}_{\gamma_{n}}\left(f_{n}\right)(u)}{u-\xi} \frac{u-z}{|u-z|} d m(u) .
\end{aligned}
$$


Donc

$$
\begin{aligned}
& \sup _{\xi \in \bar{B}(z, r)}\left|\mathcal{T}(f)(\xi)-\mathcal{T}_{\gamma_{n}}\left(f_{n}\right)(\xi)\right| \\
& \quad \leq \frac{1}{2 \pi\left(R-\rho_{1}\right)\left(\rho_{1}-r\right)} \iint_{A\left(z, \rho_{1}, R\right)}\left|\mathcal{T}(f)(u)-\mathcal{T}_{\gamma_{n}}\left(f_{n}\right)(u)\right| d m(u) .
\end{aligned}
$$

Comme $\left|\mathcal{T}(f)(u)-\mathcal{T}_{\gamma_{n}}\left(f_{n}\right)(u)\right|=\left|\mathcal{C}\left(\bar{\partial} f_{n}\right)(u)-\mathcal{C}(\bar{\partial} f)(u)\right|$ presque partout sur $A\left(z, \rho_{1}, R\right)$, il vient

$$
\sup _{\xi \in \bar{B}(z, r)}\left|\mathcal{T}(f)(\xi)-\mathcal{T}_{\gamma_{n}}\left(f_{n}\right)(\xi)\right| \leq c|| \bar{\partial} f_{n}-\bar{\partial} f \|=c|\bar{\partial} f|\left(\Omega \backslash \stackrel{\circ}{K_{n}}\right)
$$

et $\lim _{n \rightarrow \infty}|\bar{\partial} f|\left(\Omega \backslash \stackrel{\circ}{K}_{n}\right)=0$. Ceci montre que $\mathcal{T}_{\gamma_{n}}\left(f_{n}\right)$ converge uniformément vers $\mathcal{T}(f)$ sur $\bar{B}(z, r)$. Par conséquent, $\mathcal{T}_{\gamma_{n}}\left(f_{n}\right)$ converge uniformément vers $\mathcal{T}(f)$ sur tout compact de $\mathbb{C} \backslash \partial \Omega$.

Soit maintenant $B$ une partie bornée de $\mathcal{O}(\partial \Omega)$. Il existe un voisinage ouvert $U$ de $\partial \Omega$ tel que $B$ soit une partie bornée de $\mathcal{H}(U)$. Soit $V$ un voisinage ouvert de $\partial \Omega$ tel que $\bar{V} \subset U$ et tel que $\partial \bar{V}$ soit une réunion finie de courbes de Jordan rectifiables, et soit $L=\int_{\partial \bar{V}}|d z|$. Soit $g \in B$; pour $n$ assez grand, on a $\gamma_{n} \subset V$, donc

$$
\left\langle g, \mathcal{T}_{\gamma_{n}}\left(f_{n}\right)\right\rangle=-\frac{1}{2 i \pi} \int_{\partial \bar{V}} g(\xi) \mathcal{T}_{\gamma_{n}}\left(f_{n}\right)(\xi) d \xi
$$

Donc

$$
\begin{aligned}
& \left|\left\langle g, \mathcal{T}_{\gamma_{n}}\left(f_{n}\right)\right\rangle-\langle g, \mathcal{T}(f)\rangle\right| \\
& \quad \leq \frac{1}{2 \pi} \int_{\partial \bar{V}}\left|\mathcal{T}_{\gamma_{n}}\left(f_{n}\right)(\xi)-\mathcal{T}(f)(\xi)\right||g(\xi)||d \xi| \\
& \quad \leq \frac{L}{2 \pi} \sup _{h \in B}\left(\sup _{\xi \in \partial \bar{V}}|h(\xi)|\right) \sup _{\xi \in \partial \bar{V}}\left|\mathcal{T}_{\gamma_{n}}\left(f_{n}\right)(\xi)-\mathcal{T}(f)(\xi)\right| .
\end{aligned}
$$

Pour $f \in \mathcal{M}_{\text {loc }}(\Omega, \bar{\partial})$, posons $f^{*}=(\mathcal{T} f)^{+}+\mathcal{C}^{+}(\bar{\partial} f)$ et

$$
\theta(\bar{\partial} f)(z)=\frac{1}{\pi} \iint_{\Omega} \frac{d|\bar{\partial} f|(\xi)}{|z-\xi|} \in[0, \infty] \quad(z \in \mathbb{C}) .
$$

Soit $V$ un ouvert relativement compact de $\Omega$ à bord de $\operatorname{classe} \mathcal{C}^{1}$; alors $\partial V$ est une réunion finie disjointe de courbes de Jordan de classe $\mathcal{C}^{1}$. Si $\theta(\bar{\partial} f)_{\mid \partial V} \in \mathcal{L}^{1}(\partial V,|d \xi|)$, d'après le théorème de Fubini, $\mathcal{C}(\bar{\partial} f)_{\mid \partial V} \in$ $\mathcal{L}^{1}(\partial V,|d \xi|)$, donc $f_{\mid \partial V}^{*} \in \mathcal{L}^{1}(\partial V,|d \xi|)$. Notons que dans ce cas, toujours d'après le théorème de Fubini, $|\bar{\partial} f|(\partial V)=0$. 
Lemme 3.4. Soient $\Omega$ un ouvert borné de $\mathbb{C}, f \in \mathcal{M}_{\text {loc }}(\Omega, \bar{\partial})$, et $V$ un ouvert relativement compact de $\Omega$ à bord de classe $\mathcal{C}^{1}$. Si $\theta(\bar{\partial} f)_{\mid \partial V} \in$ $\mathcal{L}^{1}(\partial V,|d \xi|)$, alors $\mathcal{T}_{\partial V}\left(f_{\mid \partial V}\right)=f_{\mid \partial V}^{*}$.

Démonstration: Soit $z \in \mathbb{C} \backslash \partial V$; on a

$$
\begin{aligned}
\frac{1}{2 i \pi} \int_{\partial V} & f^{*}(\xi) R_{z}(\xi) d \xi \\
& =\frac{1}{2 i \pi} \int_{\partial V}(\mathcal{T} f)^{+}(\xi) R_{z}(\xi) d \xi+\frac{1}{2 i \pi} \int_{\partial V} \mathcal{C}^{+}(\bar{\partial} f)(\xi) R_{z}(\xi) d \xi
\end{aligned}
$$

D'une part,

$$
\frac{1}{2 i \pi} \int_{\partial V}(\mathcal{T} f)^{+}(\xi) R_{z}(\xi) d \xi= \begin{cases}(\mathcal{T} f)^{+}(z) & z \in V \\ 0 & z \notin \bar{V}\end{cases}
$$

D'autre part,

$$
\frac{1}{2 i \pi} \int_{\partial V} \mathcal{C}^{+}(\bar{\partial} f)(\xi) R_{z}(\xi) d \xi=\frac{1}{2 i \pi} \iint_{\Omega}\left(\frac{1}{2 i \pi} \int_{\partial V} \frac{R_{z}(\xi)}{\xi-\eta} d \xi\right) d(\bar{\partial} f)(\eta)
$$

car

$$
\frac{1}{2 \pi} \int_{\partial V} \theta(\bar{\partial} f)(\xi)\left|R_{z}(\xi)\right||d \xi|<\infty .
$$

De même que plus haut, on pose $R_{z}^{+}=R_{z}, R_{z}^{-}=0$ si $z \in \mathbb{C} \backslash \bar{V}$, et $R_{z}^{+}=0, R_{z}^{-}=R_{z}$ si $z \in V$. Dans tous les cas $R_{z}^{+} \in \mathcal{O}^{+}(\partial V)$, $R_{z}^{-} \in \mathcal{O}^{-}(\partial V)$ et on a, d'après la formule de Cauchy,

$$
\frac{1}{2 i \pi} \int_{\partial V} \frac{R_{z}(\xi)}{\xi-\eta} d \xi= \begin{cases}R_{z}^{+}(\eta) & \eta \in V \\ -R_{z}^{-}(\eta) & \eta \notin \bar{V}\end{cases}
$$

Donc

$$
\begin{aligned}
\frac{1}{2 i \pi} \int_{\partial V} \mathcal{C}^{+}(\bar{\partial} f)(\xi) R_{z}(\xi) d \xi= & \frac{1}{\pi} \iint_{V} R_{z}^{+}(\eta) d(\bar{\partial} f)(\eta) \\
& -\frac{1}{\pi} \iint_{\Omega \backslash V} R_{z}^{-}(\eta) d(\bar{\partial} f)(\eta) \\
& = \begin{cases}\mathcal{C}\left(\bar{\partial} f_{\mid \Omega \backslash V}\right)(z) & z \in V, \\
-\mathcal{C}\left(\bar{\partial} f_{\mid V}\right)(z) & z \notin \bar{V}\end{cases}
\end{aligned}
$$


Finalement,

$$
\begin{aligned}
\frac{1}{2 i \pi} \int_{\partial V} f^{*}(\xi) R_{z}(\xi) d \xi & = \begin{cases}f(z)-\mathcal{C}\left(\bar{\partial} f_{\mid V}\right)(z) & \text { p.p. sur } V \\
-\mathcal{C}\left(\bar{\partial} f_{\mid V}\right)(z) & z \notin \bar{V}\end{cases} \\
& =\mathcal{T}_{\partial V}\left(f_{\mid V}\right)(z) \\
& =\left\langle R_{z}, \mathcal{T}_{\partial V}\left(f_{\mid V}\right)\right\rangle .
\end{aligned}
$$

Le résultat découle alors de (2.3).

Rappelons qu'un domaine de $\mathbb{C}$ est finiment connexe (resp. $n$-connexe) si sa frontière est composée d'un nombre fini de composantes connexes (resp. de $n$ composantes connexes). Les domaines simplement connexes sont donc les domaines 1-connexes. Un domaine est circulaire lorsque sa frontière est composée d'un nombre fini de cercles disjoints, éventuellement réduits à un point. Koebe a montré en 1906 que tout domaine borné finiment connexe $\Omega$ du plan complexe est conformément équivalent à un domaine circulaire $D([\mathbf{G o}]$, et $[\mathbf{C}$, p. 106]). Soit alors $\varphi: D \rightarrow \Omega$ une représentation conforme de $\Omega$, et soient $C_{1}$ la composante connexe "externe" de $\partial D$, et $C_{2}, \ldots, C_{n}$ les composantes connexes "internes" de $\partial D$. Pour $r \in] 0,1$, notons $C_{1, r}$ le cercle obtenu en diminuant le rayon de $C_{1}$ de $1-r$, et $C_{j, r}(j=2, \ldots, n)$ le cercle obtenu en augmentant le rayon de $C_{j}$ de $1-r$. Il existe $\left.r_{0} \in\right] 0,1$ [ tel que, pour $\left.r \in\right] r_{0}, 1[$, les cercles $C_{j, r}(j=1, \ldots, n)$ soient deux à deux disjoints. Pour $\left.r \in\right] r_{0}, 1[$, posons

$$
C_{r}=\cup_{j=1}^{n} C_{j, r}
$$

et soient $D_{r} \subset D$ le domaine circulaire de frontière $C_{r}$ et $\Omega_{r}=\varphi\left(D_{r}\right)$. Si $f \in \mathcal{L}^{1}\left(\Omega \backslash \overline{\Omega_{r_{0}}}\right)$, alors $f_{\mid \partial \Omega_{r}} \in \mathcal{L}^{1}\left(\partial \Omega_{r},|d \xi|\right)$ pour presque tout $\left.r \in\right] r_{0}, 1[$. En effet, comme $\iint_{\Omega \backslash \Omega_{r_{0}}}|f(z)| d m(z)=\iint_{D \backslash D_{r_{0}}}|f(\varphi(\xi))|\left|\varphi^{\prime}(\xi)\right|^{2} d m(\xi)$, et comme $\varphi^{\prime}\left(\varphi^{-1}(z)\right)=\left[\left(\varphi^{-1}\right)^{\prime}(z)\right]^{-1} \neq 0$ pour $z \in \Omega$, on a

$$
\iint_{\Omega \backslash \Omega_{r_{0}}}|f(z)| d m(z)=\int_{r_{0}}^{1}\left[\int_{\partial \Omega_{r}}|f(z)|\left|\left(\varphi^{-1}\right)^{\prime}(z)\right|^{-1}|d z|\right] d r .
$$

En particulier, si $f \in \mathcal{M}_{\text {loc }}(\Omega, \bar{\partial})$ et si $f^{*}=\mathcal{T}(f)^{+}+\mathcal{C}^{+}(\bar{\partial} f)$, comme $f=f^{*}$ presque partout sur $\Omega$, on a $f_{\mid \partial \Omega_{r}}^{*}=f_{\mid \partial \Omega_{r}}$ pour presque tout $r \in] r_{0}, 1[$. 
Théorème 3.5. Soient $\Omega$ un domaine finiment connexe de $\mathbb{C}$ et $f \in$ $\mathcal{M}_{\mathrm{loc}}(\Omega, \bar{\partial})$. Alors

$$
\langle g, \mathcal{T} f\rangle=\lim _{r \rightarrow 1^{-}} \operatorname{ess} \frac{1}{2 i \pi} \int_{\partial \Omega_{r}} g(\xi) f(\xi) d \xi
$$

uniformément sur tout borné de $\mathcal{O}(\partial \Omega)$.

Démonstration: D'après la Proposition 3.3,

$$
\langle g, \mathcal{T} f\rangle=\lim _{r \rightarrow 1^{-}}\left\langle g, \mathcal{T}_{\partial \Omega_{r}}\left(f_{\mid \Omega_{r}}\right)\right\rangle
$$

uniformément sur tout borné de $\mathcal{O}(\partial \Omega)$. Comme $\theta(\bar{\partial} f)_{\mid \partial \Omega_{r}} \in \mathcal{L}^{1}\left(\partial \Omega_{r},|d \xi|\right)$ pour presque tout $r \in] r_{0}, 1$, d'après le Lemme 3.4,

$$
\left\langle g, \mathcal{T}_{\partial \Omega_{r}}\left(f_{\mid \Omega_{r}}\right)\right\rangle=\frac{1}{2 i \pi} \int_{\partial \Omega_{r}} g(\xi) f^{*}(\xi) d \xi=\frac{1}{2 i \pi} \int_{\partial \Omega_{r}} g(\xi) f(\xi) d \xi
$$

presque partout sur $] r_{0}, 1[$.

Supposons maintenant que $\partial \Omega$ est une réunion finie disjointe de courbes de Jordan. Soit $\varphi: D \rightarrow \Omega$ une représentation conforme où $D$ est un domaine circulaire. Alors $[\mathbf{C}$, p. 82] $\varphi$ s'étend par continuité en un homéomorphisme de $\bar{D}$ sur $\bar{\Omega}$. En particulier aucune composante de $\partial D$ n'est réduite à un point. Supposons de plus que chaque composante de $\partial \Omega$ est une courbe de Jordan rectifiable. En reprenant la démonstration d'un théorème établi par F. et M. Riesz dans le cas simplement connexe [T, p. 319], on voit que

$$
\sup _{r_{0}<r \leq 1} \int_{C_{j, r}}\left|\varphi^{\prime}(\xi)\right||d \xi|<+\infty,
$$

et que $\varphi^{\prime}$ possède des limites non tangentielles en presque tout point de $C_{j}(j=1,2, \ldots, n)$. Soit $a_{j}$ le centre de $C_{j}$ et $r_{j}$ son rayon; on a (cf. $[\mathbf{R 2}])$

$$
\begin{aligned}
& \int_{0}^{2 \pi}\left|\varphi^{\prime}\left(a_{1}+r_{1} e^{i t}\right)-\varphi^{\prime}\left(a_{1}+\rho e^{i t}\right)\right| d t \underset{\rho \rightarrow r_{1}^{-}}{\longrightarrow} 0, \\
& \int_{0}^{2 \pi}\left|\varphi^{\prime}\left(a_{j}+r_{j} e^{i t}\right)-\varphi^{\prime}\left(a_{j}+\rho e^{i t}\right)\right| d t \underset{\rho \rightarrow r_{j}^{+}}{\longrightarrow} 0 \quad(j \geq 2) .
\end{aligned}
$$

En particulier,

$$
\int_{C_{j}}\left|\varphi^{\prime}(\xi)\right||d \xi|=\lim _{r \rightarrow 1^{-}} \int_{C_{j, r}}\left|\varphi^{\prime}(\xi)\right||d \xi|=\lim _{r \rightarrow 1^{-}} L\left(\varphi\left(C_{j, r}\right)\right) \geq L\left(\varphi\left(C_{j}\right)\right),
$$


où $L(\Gamma)$ désigne la longueur de la courbe rectifiable $\Gamma$, (cf. [Tr, p. 59] pour l'inégalité ci-dessus). La fonction $\psi: t \rightarrow \varphi\left(a_{j}+r_{j} e^{i t}\right)$ est à variation bornée sur $[0,2 \pi]$. Elle est donc dérivable presque partout, et il existe une mesure singulière $\nu$ telle que

$$
\psi(t)-\psi(0)=\int_{0}^{t} \psi^{\prime}(s) d s+\nu([0, t])
$$

pour $0 \leq t \leq 2 \pi$ (cf. [R, pp. 173-177]). De plus $L\left(\varphi\left(C_{j}\right)\right)$ est la variation totale de $\psi$ sur $[0,2 \pi]$. Donc

$$
L\left(\varphi\left(C_{j}\right)\right)=\int_{0}^{2 \pi}\left|\psi^{\prime}(s)\right| d s+|\nu|([0,2 \pi]) .
$$

De même que dans [T, p. 319], on vérifie que

$$
\psi^{\prime}(t)=i r_{j} e^{i t} \varphi^{\prime}\left(a_{j}+r_{j} e^{i t}\right)
$$

presque partout, où $\varphi^{\prime}\left(a_{j}+r_{j} e^{i t}\right)$ est obtenue par limite non tangentielle de $\varphi^{\prime}$. Donc $\nu=0$,

$$
L\left(\varphi\left(C_{j}\right)\right)=\int_{C_{j}}\left|\varphi^{\prime}(\xi)\right||d \xi|
$$

et $\psi$ est absolument continue. On a donc (cf. le théorème de changement de variable [Na, p. 236])

$$
\int_{\partial \Omega} h(\xi) d \xi=\int_{\partial D} h(\varphi(\eta)) \varphi^{\prime}(\eta) d \eta \quad\left(h \in \mathcal{L}^{1}(\partial \Omega,|d \xi|)\right) .
$$

Du Théorème 3.5 on déduit alors le résultat suivant:

Corollaire 3.6. Soit $\Omega$ un domaine borné de $\mathbb{C}$ tel que $\partial \Omega$ soit une réunion finie disjointe de courbes de Jordan rectifiables.

Si $f \in \mathcal{M}_{\text {loc }}(\Omega, \bar{\partial}) \cap \mathcal{C}(\bar{\Omega})$, alors $\mathcal{T} f=f_{\mid \partial \Omega}$.

Démonstration: Soit $g \in \mathcal{O}(\partial \Omega)$. Avec les notations ci-dessus, on a

$$
\begin{aligned}
& \int_{0}^{2 \pi}\left|\varphi^{\prime}\left(a_{1}+\rho e^{i t}\right)-\varphi^{\prime}\left(a_{1}+r_{1} e^{i t}\right)\right| d t \underset{\rho \rightarrow r_{1}^{-}}{\longrightarrow} 0, \\
& \int_{0}^{2 \pi}\left|\varphi^{\prime}\left(a_{j}+\rho e^{i t}\right)-\varphi^{\prime}\left(a_{j}+r_{j} e^{i t}\right)\right| d t \underset{\rho \rightarrow r_{j}^{+}}{\longrightarrow} 0 \quad(j \geq 2) .
\end{aligned}
$$

Comme $f \circ \varphi \cdot g \circ \varphi$ est uniformément continue sur un voisinage de $\partial \Omega$ dans $\bar{\Omega}$ et comme

$$
\int_{\partial \Omega} f(\xi) g(\xi) d \xi=\int_{\partial D} f(\varphi(\eta)) g(\varphi(\eta)) \varphi^{\prime}(\eta) d \eta,
$$


un calcul simple montre que

$$
\int_{\partial \Omega} f(\xi) g(\xi) d \xi=\lim _{r \rightarrow 1^{-}} \int_{\partial \Omega_{r}} f(\xi) g(\xi) d \xi
$$

Le corollaire résulte alors directement du Théorème 3.5.

On en déduit immédiatement une formule de Cauchy-Pompeiu généralisée:

Corollaire 3.7. Soit $\Omega$ un domaine borné de $\mathbb{C}$ tel que $\partial \Omega$ soit une réunion finie disjointe de courbes de Jordan rectifiables. Si $f \in \mathcal{C}(\bar{\Omega})$, et si $\bar{\partial} f \in \mathcal{M}(\Omega)$, alors

$\frac{1}{\pi} \iint_{\Omega} \frac{d(\bar{\partial} f)(\xi)}{z-\xi}+\frac{1}{2 i \pi} \int_{\partial \Omega} \frac{f(\xi)}{\xi-z} d \xi= \begin{cases}0, & z \in \mathbb{C} \backslash \bar{\Omega} \\ f(z) & \text { pour presque tout } z \in \Omega .\end{cases}$

\section{Image de la trace de Cauchy}

Pour caractériser l'image de la trace de Cauchy, on énonce, et on démontre, deux lemmes concernant l'intégrale de Poisson (cf. [Ra] pour plus de détails).

Soient $\Omega$ un domaine borné de $\mathbb{C}$, et $f \in \mathcal{C}(\partial \Omega)$. On note $\mathcal{V}(f)$ l'ensemble des fonctions sous-harmoniques sur $\Omega$ telles que

$$
\limsup _{z \rightarrow \xi, z \in \Omega} u(z) \leq f(\xi) \quad(\xi \in \partial \Omega),
$$

et $\mathcal{U}(f)$ la fonction de Perron de $f$, définie par

$$
\mathcal{U}(f)(z)=\sup u(z) \quad(u \in \mathcal{V}(f)) .
$$

Alors, pour $z \in \Omega$, l'application $f \rightarrow \mathcal{U}(f)(z)$ est linéaire, continue et positive sur $\mathcal{C}(\partial \Omega)$, et, d'après le théorème de Riesz, il existe une unique mesure positive sur $\partial \Omega$ représentant cette forme linéaire. On l'appelle mesure harmonique de $\Omega$ en $z$, et on la note $\omega_{z}$. La fonction de Perron de $f$ est harmonique et bornée sur $\Omega$ et vérifie

$$
\lim _{z \rightarrow \xi, z \in \Omega} \mathcal{U}(f)(z)=f(\xi) \text { sur } \partial \Omega \backslash E,
$$

où $E$ est un sous-ensemble de $\partial \Omega$ de capacité logarithmique nulle.

Comme les mesures $\left\{\omega_{z}: z \in \Omega\right\}$ sont mutuellement absolument continues, les espaces $\left\{\mathcal{L}^{\infty}\left(\partial \Omega, \omega_{z}\right): z \in \Omega\right\}$ sont identiques. Fixons $z_{0} \in \Omega$, et soient $\omega=\omega_{z_{0}}$ et $f \in \mathcal{L}^{\infty}(\partial \Omega, \omega)$. L'intégrale de Poisson de $f$ est définie par la formule

$$
P(f)(z)=\int_{\partial \Omega} f(\xi) d \omega_{z}(\xi) \quad(z \in \Omega) .
$$


On note $h^{\infty}(\Omega)$ l'espace de Banach des fonctions harmoniques bornées sur $\Omega$. On a $P(f) \in h^{\infty}(\Omega)$; et $\|P(f)\|_{\infty} \leq\|f\|_{\infty}$, car $\omega_{z}$ est une mesure de probabilité.

Lemme 4.1. $P: \mathcal{L}^{\infty}(\partial \Omega, \omega) \rightarrow h^{\infty}(\Omega)$ est une isométrie.

Démonstration: Soit $f \in \mathcal{L}^{\infty}(\partial \Omega, \omega)$; si $f$ est à valeurs réelles, alors [Ra, p. 105, Exemple 2]

$$
f(\xi) \leq \limsup _{z \rightarrow \xi, z \in \Omega} P(f)(z) \quad \text { w-p.p. }
$$

En appliquant ce résultat à $-f$, on obtient $\|f\|_{\infty}=\|P(f)\|_{\infty}$.

Soit $f \neq 0$ une fonction à valeurs complexes. Soit $0<a<\|f\|_{\infty}$ et soit $U_{a}=\{z \in \partial \Omega:|f(z)|>a\}$. Pour $n \in \mathbb{N}^{*}$ et pour $0 \leq k<n$ posons

$$
U_{a, k, n}=\left\{z \in U_{a}: \frac{2 k \pi}{n} \leq \operatorname{Arg} f(z)<\frac{2(k+1) \pi}{n}\right\} .
$$

Comme $\omega\left(U_{a}\right)>0$, il existe $k$ tel que $\omega\left(U_{a, k, n}\right)>0$. Posons $g=f e^{-\frac{2 i k}{n}}$; alors $\|f\|_{\infty}=\|g\|_{\infty}$ et $\|P(f)\|_{\infty}=\|P(g)\|_{\infty}$. Si $z \in U_{a, k, n}$, on a $\operatorname{Arg} g(z) \in\left[0, \frac{2 \pi}{n}\left[\right.\right.$ donc $\operatorname{Re} g(z)>|g(z)| \cos \frac{2 \pi}{n}>a \cos \frac{2 \pi}{n}$. Comme $\omega\left(U_{a, k, n}\right)>0,\|\operatorname{Re} g\|_{\infty}>a \cos \frac{2 \pi}{n}$. Reg étant à valeurs réelles, il vient $\|\operatorname{Re} P(g)\|_{\infty}=\|P(\operatorname{Re} g)\|_{\infty}=\|\operatorname{Re} g\|_{\infty}>a \cos \frac{2 \pi}{n}$. A fortiori, $\|P(f)\|_{\infty}=\|P(g)\|_{\infty}>a \cos \frac{2 \pi}{n}$. En faisant tendre $a$ vers $\|f\|_{\infty}$ et $n$ vers l'infini, il vient $\|P(f)\|_{\infty} \geq\|f\|_{\infty}$.

Plus généralement, si $\Omega$ n'est pas connexe, soit $\left(\Omega_{n}\right)_{0 \leq n<p \leq \infty}$ la famille (finie ou dénombrable) des composantes connexes de $\Omega$. Pour $z \in \Omega$ et pour $n$ tel que $z \in \Omega_{n}$, notons $\omega_{z}$ la mesure harmonique de $\Omega_{n}$ en $z$, étendue à $\partial \Omega \operatorname{par} \omega_{z}\left(\partial \Omega \backslash \partial \omega_{n}\right)=0$. Pour $0 \leq n<p$, soit $z_{n} \in \Omega_{n}$. Associons maintenant à $\Omega$ la mesure borélienne finie définie par la formule

$$
\mu=\sum_{0 \leq n<p} \frac{\omega_{z_{n}}}{2^{n}}
$$

Les mesures $\left\{\omega_{z}: z \in \Omega\right\}$ étant absolument continues par rapport à $\mu$, on a $\omega_{z} \in \mathcal{L}^{1}(\partial \Omega, \mu)$. Soit $f \in \mathcal{L}^{\infty}(\partial \Omega, \mu)$; on définit l'intégrale de Poisson de $f$ par la formule

$$
P(f)(z)=\int_{\partial \Omega} f(\xi) d \omega_{z}(\xi) \quad(z \in \Omega) .
$$

$P(f)$ étant harmonique sur chaque composante connexe $\Omega_{n}$, elle l'est également sur $\Omega$, et

$$
\|P(f)\|_{\infty}=\sup _{0 \leq n<p}\left\|P(f)_{\mid \Omega_{n}}\right\|_{\infty}=\sup _{0 \leq n<p}\|f\|_{\mathcal{L}^{\infty}\left(\partial \Omega_{n}, \omega_{z_{n}}\right)} \leq\|f\|_{\mid \mathcal{L}^{\infty}(\partial \Omega, \mu)} .
$$


Lemme 4.2. $P: \mathcal{L}^{\infty}(\partial \Omega, \mu) \rightarrow h^{\infty}(\Omega)$ est une isométrie.

Démonstration: Soit $f$ une fonction borélienne bornée sur $\partial \Omega$. Comme $\Omega_{n}$ est une composante connexe de $\Omega$, on a $\partial \Omega_{n} \subset \partial \Omega$. Posons $V_{n}=$ $\left\{\xi \in \partial \Omega_{n}:|f(\xi)|>\|P f\|_{\infty}\right\}$. Comme $\|f\|_{\mathcal{L}^{\infty}\left(\partial \Omega_{n}, \omega_{z_{n}}\right)}=\left\|P(f)_{\mid \Omega_{n}}\right\|_{\infty}$, $\omega_{z_{n}}\left(V_{n}\right)=0$. Pour $\xi \in \cup_{0 \leq n<p}\left(\partial \Omega_{n} \backslash V_{n}\right)$, on a $|f(\xi)| \leq\|P(f)\|_{\infty}$. Il suffit donc de montrer que $\mu\left(\partial \Omega \backslash \cup_{0 \leq n<p}\left(\partial \Omega_{n} \backslash V_{n}\right)\right)=0$. Or

$$
\partial \Omega \backslash \cup_{0 \leq n<p}\left(\partial \Omega_{n} \backslash V_{n}\right)=\left(\partial \Omega \backslash \cup_{0 \leq n<p} \partial \Omega_{n}\right) \cup\left(\cap_{0 \leq n<p} V_{n}\right),
$$

$\mu\left(\partial \Omega \backslash \cup_{0 \leq n<p} \partial \Omega_{n}\right)=0$ et $\mu\left(\cap_{0 \leq n<p} V_{n}\right)=0$, donc $\|f\|_{\mathcal{L}^{\infty}(\partial \Omega, \mu)} \leq$ $\|P f\|_{\infty}$.

Remarque 4.3. Si $h \in h^{\infty}(\Omega) \cap \mathcal{C}(\bar{\Omega})$, alors $P\left(h_{\mid \partial \Omega}\right)=h$. En effet, pour tout $n, P\left(h_{\mid \partial \Omega}\right)_{\mid \Omega_{n}}$ et $h_{\mid \Omega_{n}}$ sont harmoniques sur $\Omega_{n}$. Comme $h_{\mid \overline{\Omega_{n}}} \in \mathcal{C}\left(\overline{\Omega_{n}}\right)$, on a $\lim _{z \rightarrow \xi, z \in \Omega_{n}} h(z)=h(\xi)$ sur $\partial \Omega_{n}$. D'autre part, soit $z \in \Omega_{n}$. Comme $\omega_{z}\left(\partial \Omega \backslash \partial \Omega_{n}\right)=0$, on a

$$
P\left(h_{\mid \partial \Omega}\right)(z)=\int_{\partial \Omega_{n}} h(\xi) d \omega_{z}(\xi) \quad\left(z \in \Omega_{n}\right) .
$$

Comme $h \in \mathcal{C}\left(\partial \Omega_{n}\right)$, on a

$$
\lim _{z \rightarrow \xi, z \in \Omega_{n}} P\left(h_{\mid \partial \Omega}\right)(z)=h(\xi) \quad\left(\xi \in \partial \Omega_{n} \backslash E\right),
$$

où $E$ est un sous-ensemble de $\partial \Omega_{n}$ de capacité logarithmique nulle. Par unicité de la solution du problème de Dirichlet généralisé (cf. [Ra, p. 95]), il vient $P\left(h_{\mid \partial \Omega}\right)_{\mid \Omega_{n}}=h_{\mid \Omega_{n}}$. Donc $P\left(h_{\mid \partial \Omega}\right)=h$.

Théorème 4.4. Soit $\Omega$ un ouvert borné de $\mathbb{C}$, et soit $\mu=\sum_{0 \leq n<p} \frac{\omega_{z_{n}}}{2^{n}}$. Alors

$$
\mathcal{C}^{-}(\mathcal{M}(\Omega))=\mathcal{C}^{-}\left(\mathcal{L}^{1}(\Omega)\right)=\mathcal{P}^{-}\left(\mathcal{L}^{1}(\partial \Omega, \mu)\right),
$$

où $\mathcal{P}^{-}(f)(z)=\frac{1}{2 i \pi} \int_{\partial \Omega} \frac{f(\xi)}{\xi-z} d \mu(\xi)(z \notin \bar{\Omega})$ pour $f \in \mathcal{L}^{1}(\partial \Omega, \mu)$.

Démonstration: L'application $z \rightarrow \omega_{z}, \Omega \rightarrow \mathcal{L}^{1}(\partial \Omega, \mu)$ est continue. En effet, soient $z, z^{\prime} \in \Omega_{n}(0 \leq n<p)$; on a

$$
\begin{aligned}
\left\|\omega_{z}-\omega_{z^{\prime}}\right\| & =\sup _{h \in \mathcal{C}(\partial \Omega),\|h\| \leq 1}\left|\int_{\partial \Omega} h(\xi) d \omega_{z}(\xi)-\int_{\partial \Omega} h(\xi) d \omega_{z^{\prime}}(\xi)\right| \\
& =\sup _{h \in \mathcal{C}(\partial \Omega),\|h\| \leq 1}\left|P(h)(z)-P(h)\left(z^{\prime}\right)\right| .
\end{aligned}
$$


Les opérateurs $\frac{\partial}{\partial x}$ et $\frac{\partial}{\partial y}$ étant continus sur $\mathcal{C}^{\infty}(\Omega)$ et, d'après le théorème du graphe fermé, $P: \mathcal{C}(\partial \Omega) \rightarrow \mathcal{C}^{\infty}(\Omega)$ étant continue, pour tout compact $K$ de $\Omega_{n}$, il existe une constante $c_{K}$ telle que

$$
\sup _{z \in K}\left(\left|\frac{\partial P(h)}{\partial x}(z)\right|+\left|\frac{\partial P(h)}{\partial y}(z)\right|\right) \leq c_{K}\|h\|_{\infty} .
$$

Par le théorème des accroissements finis, il vient donc $\left\|\omega_{z}-\omega_{z^{\prime}}\right\| \leq$ $c_{K}\left|z-z^{\prime}\right|$.

On pose

$$
S(\nu)=\iint_{\Omega} \omega_{z} d \nu(z) \quad(\nu \in \mathcal{M}(\Omega)) .
$$

Comme $\left\|\omega_{z}\right\|_{\mathcal{L}^{1}(\partial \Omega, \mu)}=1$, l'opérateur $S: \mathcal{M}(\Omega) \rightarrow \mathcal{L}^{1}(\partial \Omega, \mu)$ est une contraction. Posons $\langle f, g\rangle=\int_{\partial \Omega} f(\xi) g(\xi) d \mu(\xi)$ pour $f \in \mathcal{L}^{\infty}(\partial \Omega, \mu)$ et $g \in \mathcal{L}^{1}(\partial \Omega, \mu)$. On a

$$
\begin{aligned}
& \iint_{\Omega} P(f)(z) d \nu(z) \\
= & \iint_{\Omega}\left\langle f, \omega_{z}\right\rangle d \nu(z) \\
= & \left\langle f, \iint_{\Omega} \omega_{z} d \nu(z)\right\rangle \\
= & \int_{\partial \Omega} S(\nu)(z) f(z) d \mu(z) \quad\left(f \in \mathcal{L}^{\infty}(\partial \Omega, \mu), \nu \in \mathcal{M}(\Omega)\right) .
\end{aligned}
$$

Pour $z \in \mathbb{C} \backslash \bar{\Omega}, R_{z} \in h^{\infty}(\Omega) \cap \mathcal{C}(\bar{\Omega})$ et $P\left(R_{z \mid \partial \Omega}\right)=R_{z}$, donc

$$
\begin{aligned}
\mathcal{C}^{-}(\nu)(z) & =-\frac{1}{\pi} \iint_{\Omega} P\left(R_{z \mid \partial \Omega}\right)(\xi) d \nu(\xi) \\
& =-\frac{1}{\pi} \int_{\partial \Omega} S(\nu)(\xi) R_{z}(\xi) d \mu(\xi)=\mathcal{P}^{-}(-2 i S(\nu))(z) .
\end{aligned}
$$

Il suffit donc de montrer que

$$
S\left(\mathcal{L}^{1}(\Omega)\right)=S(\mathcal{M}(\Omega))=\mathcal{L}^{1}(\partial \Omega, \mu) .
$$

Les inclusions $S\left(\mathcal{L}^{1}(\Omega)\right) \subset S(\mathcal{M}(\Omega)) \subset \mathcal{L}^{1}(\partial \Omega, \mu)$ sont évidentes. Pour $z \in \Omega$, et pour $\rho>0$ tel que $B(z, \rho) \subset \Omega$, posons $\varphi=\frac{1}{\pi \rho^{2}} \chi_{B(z, \rho)}$; alors $\varphi \in \mathcal{L}^{1}(\Omega)$ et $S(\varphi)=\omega_{z}$, donc

$$
\operatorname{Lin}\left\{\omega_{z}: z \in \Omega\right\} \subset S\left(\mathcal{L}^{1}(\Omega)\right) .
$$


De plus, si $f \in \mathcal{L}^{\infty}(\partial \Omega, \mu)$ et si $\int_{\partial \Omega} f d \omega_{z}=0$ pour $z \in \Omega$, alors $P(f)=0$, donc $f=0$ et, par le théorème de Hahn-Banach,

$$
\operatorname{Span}\left\{\omega_{z}: z \in \Omega\right\}=\mathcal{L}^{1}(\partial \Omega, \mu) .
$$

Il suffit donc de montrer que $S\left(\mathcal{L}^{1}(\Omega)\right)$ est fermé dans $\mathcal{L}^{1}(\partial \Omega, \mu)$. En fait, on va voir que l'application

$$
\begin{aligned}
i: \mathcal{L}^{1}(\Omega) / \operatorname{Ker} S \cap \mathcal{L}^{1}(\Omega) & \longrightarrow \mathcal{L}^{1}(\partial \Omega, \mu) \\
{[G] } & \longmapsto S(G)
\end{aligned}
$$

est une isométrie. D'après (4.3),

$$
\begin{aligned}
\text { Ker } S \cap \mathcal{L}^{1}(\Omega)={ }^{\perp} P\left(\mathcal{L}^{\infty}(\partial \Omega, \mu)\right) \\
\quad:=\left\{F \in L^{1}(\Omega): \iint_{\Omega} F(z) P(f)(z) d m(z)=0\left(f \in \mathcal{L}^{\infty}(\partial \Omega, \mu)\right)\right\}
\end{aligned}
$$

et $P: \mathcal{L}^{\infty}(\partial \Omega, \mu) \rightarrow \mathcal{L}^{\infty}(\Omega)$ est $w^{*}$-continue. Soit $\left(f_{n}\right)_{n \geq 1}$ une suite de $\mathcal{L}^{\infty}(\partial \Omega, \mu)$ telle que $P\left(f_{n}\right) \underset{n \rightarrow \infty}{\stackrel{w^{*}}{\longrightarrow}} F \in \mathcal{L}^{\infty}(\Omega)$. On a $\left\|f_{n}\right\|_{\infty}=$ $\left\|P\left(f_{n}\right)\right\|_{\infty}$. Comme la suite $\left(f_{n}\right)_{n>1}$ est bornée, on peut en extraire une sous-suite $\left(f_{n_{p}}\right)_{p \geq 1} w^{*}$-convergente vers $f \in \mathcal{L}^{\infty}(\partial \Omega, \mu)$. Par conséquent $P\left(f_{n_{p}}\right) \underset{p \rightarrow \infty}{\stackrel{w^{*}}{\longrightarrow}} P(f)$ et $F=P(f)$. Donc le convexe $P\left(\mathcal{L}^{\infty}(\partial \Omega, \mu)\right)$ est $w^{*}$-séquentiellement fermé dans $\mathcal{L}^{\infty}(\Omega)$ et, puisque $\mathcal{L}^{1}(\Omega)$ est séparable, il résulte du théorème de Krein-Šmulian [B, p. 53] que $P\left(\mathcal{L}^{\infty}(\partial \Omega, \mu)\right)$ est $w^{*}$-fermé dans $\mathcal{L}^{\infty}(\Omega)$. Donc le dual de $\mathcal{L}^{1}(\Omega) / \operatorname{Ker} S \cap \mathcal{L}^{1}(\Omega)$ est isométriquement isomorphe à $P\left(\mathcal{L}^{\infty}(\partial \Omega, \mu)\right)$, et on voit que pour $G \in \mathcal{L}^{1}(\Omega)$

$$
\begin{aligned}
\|S(G)\| & =\sup _{f \in \mathcal{L}^{\infty}(\partial \Omega, \mu),\|f\| \leq 1}\left|\int_{\partial \Omega} f S(G) d \mu\right| \\
& =\sup _{F \in P\left(\mathcal{L}^{\infty}(\partial \Omega, \mu)\right),\|F\|_{\infty} \leq 1}\left|\iint_{\Omega} F G d m\right|=\|[G]\|,
\end{aligned}
$$

donc $i$ est bien une isométrie.

Notons $\mathcal{L}_{\text {loc }}^{1}(\Omega, \bar{\partial})=\left\{f \in \mathcal{L}_{\text {loc }}^{1}(\Omega): \bar{\partial} f \in \mathcal{L}^{1}(\Omega)\right\}$.

Corollaire 4.5. Soit $\Omega$ un ouvert borné de $\mathbb{C}$ et soit $\mu=\sum_{0 \leq n<p} \frac{\omega_{z_{n}}}{2^{n}}$; on $a$

$$
\mathcal{T}\left(\mathcal{M}_{\text {loc }}(\Omega, \bar{\partial})\right)=\mathcal{T}\left(\mathcal{L}_{\text {loc }}^{1}(\Omega, \bar{\partial})\right)=\mathcal{H}(\Omega) \oplus \mathcal{P}^{-}\left(\mathcal{L}^{1}(\partial \Omega, \mu)\right) .
$$


Démonstration: Il est clair que $\mathcal{T}\left(\mathcal{L}_{\text {loc }}^{1}(\Omega, \bar{\partial})\right) \subset \mathcal{T}\left(\mathcal{M}_{\text {loc }}(\Omega, \bar{\partial})\right) \subset \mathcal{H}(\Omega) \oplus$ $\mathcal{P}^{-}\left(\mathcal{L}^{1}(\partial \Omega, \mu)\right)$. Réciproquement, soit $f \in \mathcal{H}(\Omega)$; alors $f \in \mathcal{L}_{\text {loc }}^{1}(\Omega, \bar{\partial})$ et $\mathcal{T}(f)=(f, 0)$. De même, soit $g \in \mathcal{P}^{-}\left(\mathcal{L}^{1}(\partial \Omega, \mu)\right)$; d'après le théorème précèdent, il existe $G \in \mathcal{L}^{1}(\Omega)$ tel que $-\mathcal{C}^{-}(G)=g$. Posons $h=\mathcal{C}^{+}(G)$; alors $h \in \mathcal{L}_{\text {loc }}^{1}(\Omega, \bar{\partial})$ et $\mathcal{T}(h)=\left(h-\mathcal{C}^{+}(\bar{\partial} h),-\mathcal{C}^{-}(\bar{\partial} h)\right)=(0, g)$. Donc $\mathcal{H}(\Omega) \oplus \mathcal{P}^{-}\left(\mathcal{L}^{1}(\partial \Omega, \mu)\right) \subset \mathcal{T}\left(\mathcal{L}_{\text {loc }}^{1}(\Omega, \bar{\partial})\right)$.

Remarques 4.6.

(1) Supposons que la frontière de $\Omega$ soit une réunion finie disjointe de courbes de Jordan rectifiables, et soit $\varphi: D \rightarrow \Omega$ une représentation conforme de $\Omega$ sur un domaine circulaire $D$. Comme $\partial D$ est réelle analytique, il résulte de [BG1, Proposition 4.7.21, p. 407], que la mesure harmonique $\omega_{z}$ et la mesure longueur d'arc sont mutuellement absolument continues sur $\partial D$ pour $z \in D$. Il résulte d'autre part de [Ga, Lemme 4.3, p. 149], que pour $h \in \mathcal{L}^{1}\left(d \omega_{z}\right)(z \in \Omega)$, on a

$$
\int_{\partial \Omega} h(\xi) d \omega_{z}(\xi)=\int_{\partial D} h(\varphi(\eta)) d \omega_{\varphi^{-1}(z)}(\eta) .
$$

Comme $\varphi_{\mid \partial D}$ est absolument continue, on a (cf. (3.3))

$$
\int_{\partial \Omega} h(\xi)|d \xi|=\int_{\partial D} h(\varphi(\eta))\left|\varphi^{\prime}(\eta)\right||d \eta| \quad\left(h \in \mathcal{L}^{1}(|d \xi|)\right) .
$$

Comme $\varphi^{\prime}(\eta) \neq 0$ presque partout sur $\partial D$ (cf. [T, p. 218]), et comme d'après $(3.2) \varphi_{\mid \partial D}^{\prime} \in \mathcal{L}^{1}(\partial D,|d \eta|)$, on en déduit que $\omega_{z}$ et la mesure longueur d'arc sont mutuellement absolument continues sur $\partial \Omega$. On a donc dans ce cas

$$
\mathcal{C}^{-}(\mathcal{M}(\Omega))=\mathcal{P}^{-}\left(\mathcal{L}^{1}(\partial \Omega,|d \xi|)\right)
$$

(où l'on remplace le $\mu$ de la définition de $\mathcal{P}^{-}$par $|d \xi|$ ), qui est un résultat bien connu pour le cas du disque unité $\mathbb{D}[\mathbf{N}],[\mathbf{S}]$.

(2) Soit $U$ un domaine simplement connexe dont la frontière est une courbe de Jordan rectifiable régulière au sens d'Ahlfors, c'est-à-dire telle qu'il existe $c \geq 0$ vérifiant $L\left(\partial U \cap B\left(z_{0}, r\right)\right) \leq c r$ pour tout $r>0$ et tout $z_{0} \in \mathbb{C}($ cf. $[\mathbf{D}])$. Pour $f \in \mathcal{L}^{1}(\partial U,|d \xi|)$, posons

$$
\begin{array}{ll}
P^{+}(f)(z)=\frac{1}{2 i \pi} \int_{\partial U} \frac{f(\xi)}{\xi-z} d \xi & (z \in U), \\
P^{-}(f)(z)=\frac{1}{2 i \pi} \int_{\partial U} \frac{f(\xi)}{\xi-z} d \xi & (z \notin \bar{U}) .
\end{array}
$$

Il résulte d'un théorème de Privalov [P, pp. 192-194], que $P^{+}(f)$ et $P^{-}(f)$ admettent des limites non tangentielles presque partout sur $\partial U$ 
et que $f(\xi)=P^{+}(f)(\xi)-P^{-}(f)(\xi)$ presque partout sur $\partial U$. Notons $\mathcal{L}_{0}^{1, \infty}(\partial U)$ l'ensemble des fonctions $g$ mesurables sur $\partial U$ telles que $L\left(E_{a}\right)=o\left(\frac{1}{a}\right), a \rightarrow \infty$, où $E_{a}=\{\xi \in \partial U:|g(\xi)|>a\}$. Une fonction $g \in \mathcal{L}_{0}^{1, \infty}(\partial U)$ est dite $(A)$-intégrable si $\int_{\partial U \backslash E_{a}} g(\xi) d \xi$ admet une limite quand $a$ tend vers l'infini. Cette limite est alors notée $\int_{\partial U}^{(A)} g(\xi) d \xi$. Il résulte de théorèmes de Salimov [Sa, Théorèmes 6 et $6^{\prime}$ ], qui étendent des travaux antérieurs d'Ul'janov $[\mathbf{U}]$ que si $f \in \mathcal{L}^{1}(\partial U,|d \xi|)$, les fonctions $\xi \rightarrow P^{+}(f)(\xi)$ et $\xi \rightarrow P^{-}(f)(\xi)$ sont $(A)$-intégrables sur $\partial U$, et que l'on a

$$
\begin{aligned}
& \frac{1}{2 i \pi} \int_{\partial U}^{(A)} \frac{P^{+}(f)(\xi)}{\xi-z} d \xi=\left\{\begin{array}{ll}
P^{+}(f)(z) & (z \in U) \\
0 & (z \notin \bar{U})
\end{array},\right. \\
& \frac{1}{2 i \pi} \int_{\partial U}^{(A)} \frac{P^{-}(f)(\xi)}{\xi-z} d \xi=\left\{\begin{array}{ll}
0 & (z \in U) \\
-P^{-}(f)(z) & (z \notin \bar{U})
\end{array} .\right.
\end{aligned}
$$

Soit maintenant $\Omega$ un domaine dont la frontière est une réunion finie disjointe de courbes de Jordan rectifiables Ahlfors-régulières. On pose pour $f \in \mathcal{L}^{1}(\partial \Omega,|d z|), z \notin \bar{\Omega}$,

$$
P^{-}(f)(z)=\frac{1}{2 i \pi} \int_{C_{1}} \frac{f(\xi)}{\xi-z} d \xi-\sum_{i=2}^{n} \frac{1}{2 i \pi} \int_{C_{i}} \frac{f(\xi)}{\xi-z} d \xi,
$$

$C_{1}$ désignant la frontière de la composante connexe non bornée de $\mathbb{C} \backslash \bar{\Omega}$, et $C_{2}, \ldots, C_{n}$ désignant les autres composantes connexes de $\partial \Omega$. Il résulte alors du théorème de Privalov que $P^{-}(f)$ admet des limites non tangentielles presque partout sur $\partial \Omega$, et il résulte du théorème de Salimov que

$$
P^{-}(f)(z)=-\frac{1}{2 i \pi} \int_{\partial \Omega}^{(A)} \frac{P^{-}(f)(\xi)}{\xi-z} d \xi \quad(z \in \mathbb{C} \backslash \bar{\Omega}) .
$$

On voit donc que si $\nu \in \mathcal{M}(\Omega)$ on peut intérpréter $\mathcal{T}\left(\mathcal{C}^{+}(\nu)\right)=$ $\left(0,-\mathcal{C}^{-}(\nu)\right) \in \mathcal{O}^{\prime}(\partial \Omega)$ comme la fonction définie presque partout sur $\partial \Omega$ par la formule

$$
\mathcal{T}\left(\mathcal{C}^{+}(\nu)\right)(\xi):=-\operatorname{limn} \text {.t. }{ }_{z \rightarrow \xi, z \notin \bar{\Omega}} \frac{1}{\pi} \iint_{\Omega} \frac{d \nu(\eta)}{z-\eta}
$$

$\left(\operatorname{car} \mathcal{C}^{-}(\nu)=P^{-}(f)\right.$ avec $\left.f \in \mathcal{L}^{1}(d \Omega,|d \xi|)\right)$, et on a alors

$$
\mathcal{T}\left(\mathcal{C}^{+}(\nu)\right)(z)=-\frac{1}{2 i \pi} \int_{\partial \Omega}^{(A)} \frac{\mathcal{T}\left(\mathcal{C}^{+}(\nu)\right)(\xi)}{\xi-z} d \xi \quad(z \notin \partial \Omega),
$$

la $(A)$-intégrale ci-dessus étant nulle pour $z \in \Omega$. 


\section{Noyau de la trace de Cauchy}

Soit $\Omega$ un ouvert borné de $\mathbb{C}$, et soit $\mathcal{M}_{c}(\Omega, \bar{\partial})$ l'ensemble des fonctions $f \in \mathcal{M}_{\text {loc }}(\Omega, \bar{\partial})$ à support compact dans $\Omega$; on a

$$
\mathcal{M}_{c}(\Omega, \bar{\partial}) \subset \operatorname{Ker} \mathcal{T} \subset \mathcal{L}^{1}(\Omega) .
$$

En effet, d'une part l'inclusion $\operatorname{Ker} \mathcal{T} \subset \mathcal{L}^{1}(\Omega)$ est évidente; d'autre part, notons $\tilde{f}$ la prolongée de $f$ à $\mathbb{C}$ nulle sur $\mathbb{C} \backslash \Omega$; si $f \in \mathcal{M}_{c}(\Omega, \bar{\partial})$, alors $\bar{\partial} \tilde{f}=\widetilde{\bar{\partial} f} ; \operatorname{donc} \mathcal{C}(\bar{\partial} f)=\tilde{f}$ et $\mathcal{T} f=0$.

Un exemple intéressant de fonctions de $\mathcal{M}_{\text {loc }}(\mathbb{D}, \bar{\partial})$ de trace nulle est donné par les séries de Wolff-Denjoy, c'est-à-dire les séries de la forme $\sum_{k=1}^{\infty} \frac{A_{k}}{z-\lambda_{k}}$, avec $\sum_{k=1}^{\infty}\left|A_{k}\right|<+\infty, \lambda_{k} \in \mathbb{D}$, qui s'annulent pour $|z|>1$. En effet, si on pose $\mu=\sum_{k=1}^{\infty} \pi A_{k} \delta_{\lambda_{k}}$, on a $\mathcal{C}(\mu)(z)=$ $\sum_{k=1}^{\infty} \frac{A_{k}}{z-\lambda_{k}}$ pour $z \notin \overline{\left\{\lambda_{k}\right\}_{k \geq 1}}$. Donc si on pose $f(z)=\sum_{k=1}^{\infty} \frac{A_{k}}{z-\lambda_{k}}$ pour $|z|<1, z \notin\left\{\lambda_{k}\right\}_{k \geq 1}$, on a $f \in \mathcal{M}_{\text {loc }}(\mathbb{D}, \bar{\partial}), \bar{\partial} f=\mu$ et $\mathcal{T}(f)=0$. Nous renvoyons à $[\mathbf{S}]$ pour une description détaillée des séries de Wolff-Denjoy générales.

On note $v_{c}$ (resp. $v$, resp. $v_{\infty}$ ) la topologie de la convergence simple sur $\mathcal{C}_{c}(\Omega)$ (resp. $\mathcal{C}(\bar{\Omega})$, resp. $\mathcal{C}_{b}(\Omega)$ ) où $\mathcal{C}_{b}(\Omega)$ désigne l'ensemble des fonctions continues bornées sur $\Omega$. On note également $\|\cdot\|_{1}$ la norme $\mathcal{L}^{1}$ et $\|\cdot\|$ la norme de $\mathcal{M}(\Omega)$. On cherche à déterminer des conditions nécessaires ou suffisantes sur $\Omega$ telles que $\mathcal{M}_{c}(\Omega, \bar{\partial})$ soit dense dans $\operatorname{Ker} \mathcal{T}$ pour la topologie $\left(v_{c}, v\right)$ (resp. $\left(v_{c}, v_{\infty}\right)$, resp. $\left.\left(\|\cdot\|_{1},\|\cdot\|\right)\right)$.

On note $\mathcal{R}(\bar{\Omega})$ l'adhérence uniforme dans $\mathcal{C}(\bar{\Omega})$ des fonctions rationnelles à pôles hors de $\bar{\Omega}, \mathcal{A}(\Omega)$ l'algèbre des fonctions analytiques sur $\Omega$, continues sur $\bar{\Omega}$, et $\mathcal{H}^{\infty}(\Omega)$ l'algèbre des fonctions analytiques bornées sur $\Omega$. On a $\mathcal{R}(\bar{\Omega}) \subset \mathcal{A}(\Omega) \subset \mathcal{H}^{\infty}(\Omega) \subset \mathcal{L}^{\infty}(\Omega)$ et $\mathcal{H}^{\infty}(\Omega)$ est $w^{*}$-fermé dans $\mathcal{L}^{\infty}(\Omega)$. Par la formule de Cauchy, et en considérant les sommes de Riemann, on vérifie que l'on a la propriété bien connue

$$
\operatorname{Span}\left\{R_{z}: z \notin \bar{\Omega}\right\}=\mathcal{R}(\bar{\Omega}) .
$$

Proposition 5.1. Soit $\Omega$ un ouvert borné de $\mathbb{C} ;$ alors $\mathcal{T}: \mathcal{M}_{\text {loc }}(\Omega, \bar{\partial}) \rightarrow$ $\mathcal{F}(\partial \Omega)$ est $\left(v_{c}, v\right)-w$-continue, $w$ désignant la topologie duale faible sur $\mathcal{F}(\partial \Omega)$.

Démonstration: Soit $g \in \mathcal{O}(\partial \Omega)$, et soit $g=g^{+}+g^{-}$la décomposition canonique de $g$, avec $g^{+} \in \mathcal{O}^{+}(\partial \Omega)$ et $g^{-} \in \mathcal{O}^{-}(\partial \Omega)$. Il existe un voisinage $U$ de $\partial \Omega$ tel que $g^{+} \in \mathcal{H}(\Omega \cup U)$ et $g^{-} \in \mathcal{H}_{0}((\mathbb{C} \backslash \bar{\Omega}) \cup U)$. Soit $V$ un ouvert tel que $\partial \Omega \subset V \subset \bar{V} \subset U$ et tel que $\partial V$ soit une réunion finie disjointe de courbes de Jordan de classe $\mathcal{C}^{1}$, et soit $f \in \mathcal{M}_{\text {loc }}(\Omega, \bar{\partial})$; 
d'après (2.7), on a

$$
\langle g, \mathcal{T} f\rangle=\left\langle g^{-}, \mathcal{T} f^{+}\right\rangle+\left\langle g^{+}, \mathcal{T} f^{-}\right\rangle .
$$

Pour $z \notin \bar{\Omega}$, on a

$$
\left\langle R_{z}, \mathcal{T} f^{-}\right\rangle=\mathcal{T} f^{-}(z)=\frac{1}{\pi} \iint_{\Omega} \frac{d(\bar{\partial} f)(\xi)}{\xi-z},
$$

donc, puisque $\operatorname{Span}\left\{R_{z}: z \notin \bar{\Omega}\right\}=\mathcal{O}(\bar{\Omega})=\mathcal{O}^{+}(\partial \Omega)$, on a

$$
\left\langle g^{+}, \mathcal{T} f^{-}\right\rangle=\frac{1}{\pi} \iint_{\Omega} g^{+}(\xi) d(\bar{\partial} f)(\xi) .
$$

Soit $\varphi$ une fonction de classe $\mathcal{C}^{\infty}$ à support contenu dans $\Omega \cap U$ tel que $\varphi \equiv 1$ sur un voisinage de $\partial V \cap \Omega$. Alors $\varphi g^{-}$est de classe $\mathcal{C}^{\infty}$ sur $\mathbb{C}$. On a

$$
\left\langle g^{-}, \mathcal{T} f^{+}\right\rangle=-\frac{1}{2 i \pi} \int_{\partial V \cap \Omega}\left(\varphi g^{-}\right)(\xi) \mathcal{T} f^{+}(\xi) d \xi
$$

On a $\partial V \cap \Omega=\partial \complement \bar{V} \cap \Omega$, et l'orientation canonique de $\partial \complement \bar{V} \cap \Omega$ est l'opposée de celle de $\partial V \cap \Omega$ dans la formule ci-dessus. D'après la formule de Stokes,

$$
\left\langle g^{-}, \mathcal{T} f^{+}\right\rangle=\frac{1}{\pi} \iint_{C \bar{V} \cap \Omega} \bar{\partial}\left(\varphi g^{-}\right)(\xi) \mathcal{T} f^{+}(\xi) d m(\xi) .
$$

Posons $\psi=\bar{\partial}\left(\varphi g^{-}\right) \cdot \chi_{\mathrm{C} \bar{V} \cap \Omega} ;$ alors $\psi \in \mathcal{C}_{c}^{\infty}(\mathbb{C})$ et $\operatorname{Supp} \psi \subset \Omega$. On a alors

$$
\left\langle g^{-}, \mathcal{T} f^{+}\right\rangle=\frac{1}{\pi} \iint_{\Omega} \psi(\xi) \mathcal{T} f^{+}(\xi) d m(\xi) .
$$

Par le théorème de Fubini,

$$
-\frac{1}{\pi} \iint_{\Omega} \mathcal{C}^{+}(\bar{\partial} f)(\xi) \psi(\xi) d m(\xi)=\frac{1}{\pi} \iint_{\Omega} \mathcal{C}^{+}(\psi)(\xi) d(\bar{\partial} f)(\xi) .
$$

Donc

$$
\begin{aligned}
\langle g, \mathcal{T} f\rangle= & \frac{1}{\pi} \iint_{\Omega} g^{+}(\xi) d(\bar{\partial} f)(\xi) \\
& +\frac{1}{\pi} \iint_{\Omega} \psi(\xi) f(\xi) d m(\xi) \\
& +\frac{1}{\pi} \iint_{\Omega} \mathcal{C}^{+}(\psi)(\xi) d(\bar{\partial} f)(\xi) .
\end{aligned}
$$

Comme $\mathcal{C}^{+}(\psi) \in \mathcal{C}^{\infty}(\bar{\Omega})$, ceci achève la démonstration.

Proposition 5.2. Si $\mathcal{M}_{c}(\Omega, \bar{\partial})$ est $\left(v_{c}, v_{\infty}\right)$-dense (resp. $\left(v_{c}, v\right)$-dense) dans $\operatorname{Ker} \mathcal{T}$, alors $\mathcal{R}(\bar{\Omega})$ est $w^{*}$-dense dans $\mathcal{H}^{\infty}(\Omega)$ (resp. $\mathcal{A}(\Omega)$ ). 
Démonstration: Supposons qu'il existe $h \in \mathcal{H}^{\infty}(\Omega)(\operatorname{resp} . \mathcal{A}(\Omega))$ et $f \in$ $\mathcal{L}^{1}(\Omega)$ tels que $f \perp \mathcal{R}(\bar{\Omega})$ et $\iint_{\Omega} h(z) f(z) d m(z) \neq 0$. Soit l'application

$$
\begin{aligned}
L_{h}: \mathcal{M}_{\mathrm{loc}}(\Omega, \bar{\partial}) & \longrightarrow \mathbb{C} \\
g & \longrightarrow \iint_{\Omega} h(z) d(\bar{\partial} g)(z) .
\end{aligned}
$$

Alors $L_{h}$ est $\left(v_{c}, v_{\infty}\right)$-continue (resp. $\left(v_{c}, v\right)$-continue). Soit $\varphi=\mathcal{C}^{+}(f)$; on a $\varphi \in \operatorname{Ker} \mathcal{T}$ et $L_{h}(\varphi) \neq 0$. Par contre, pour $g \in \mathcal{M}_{c}(\Omega, \bar{\partial})$, soit $\theta \in \mathcal{C}_{c}^{\infty}(\Omega)$ tel que $\theta \equiv 1$ au voisinage de $\operatorname{Supp} g$. On a

$$
\begin{aligned}
L_{h}(g) & =\iint_{\Omega}(\theta h)(\xi) d(\bar{\partial} g)(\xi) \\
& =-\iint_{\Omega} g(\xi)[h(\xi) \bar{\partial} \theta(\xi)+\theta(\xi) \bar{\partial} h(\xi)] d m(\xi)=0 .
\end{aligned}
$$

Dans la proposition qui suit, on donne quelques exemples d'ouverts $\Omega$ pour lesquels $\mathcal{R}(\bar{\Omega})$ n'est pas $w^{*}$-dense dans $\mathcal{A}(\Omega)$ ou $\mathcal{H}^{\infty}(\Omega)$. Les deux premiers sont évidents, le dernier est plus sophistiqué.

\section{Proposition 5.3.}

(I) Soit $\Omega=\mathbb{D} \backslash\left[-\frac{1}{2}, \frac{1}{2}\right]$; alors $\mathcal{R}(\bar{\Omega})$ n'est pas $w^{*}$-dense dans $\mathcal{H}^{\infty}(\Omega)$.

(II) Soit $\mathcal{C}$ le Cantor triadique, $J=\frac{1}{2} \mathcal{C} \times \frac{1}{2} \mathcal{C}$ et $\Omega=\mathbb{D} \backslash J$; alors $\mathcal{R}(\bar{\Omega})$ n'est pas $w^{*}$-dense dans $\mathcal{A}(\Omega)$.

(III) Soit $\left(\Delta_{n}\right)_{n \geq 1}$ une suite de disques fermés de $\mathbb{D}$, deux à deux disjoints, disjoints de $J$, s'accumulant exactement sur l'ensemble $J$ et qui satisfont $\sum_{n=1}^{\infty} r_{n}<\infty$, où $r_{n}$ est le rayon de $\Delta_{n}$. Posons $\Omega=\mathbb{D} \backslash\left(J \cup\left(\cup_{n \geq 1} \Delta_{n}\right)\right)$. Alors $\Omega$ est connexe, $\Omega=\stackrel{\circ}{\bar{\Omega}}$ et $\mathcal{R}(\bar{\Omega})$ n'est pas $w^{*}$-dense dans $\mathcal{A}(\Omega)$.

Démonstration:

(I) On a $\mathcal{R}(\bar{\Omega})=\mathcal{R}(\overline{\mathbb{D}})=\mathcal{A}(\mathbb{D})$. Notons $\gamma(E)$ la capacité analytique de $E \subset \mathbb{C}$ (cf. [Ga, p. 195]). Comme $\gamma\left(\left[-\frac{1}{2}, \frac{1}{2}\right]\right)=\frac{1}{4} \neq 0$, il existe $f \in$ $\mathcal{H}^{\infty}(\Omega) \backslash \mathcal{H}^{\infty}(\mathbb{D})$. Comme $\mathcal{H}^{\infty}(\mathbb{D})$ est $w^{*}$-fermé dans $\mathcal{L}^{\infty}(\Omega)=\mathcal{L}^{\infty}(\mathbb{D})$, $f$ n'appartient pas à la $w^{*}$-adhérence de $\mathcal{R}(\bar{\Omega})$.

(II) On a $m(J)=0$. Notons $\alpha(J)$ la capacité analytique continue de $J$ [Ga, p. 203]. Comme $\alpha(J) \neq 0$ (cf. [Ga, p. 238, Exemple 8]), il existe $F \in \mathcal{H}_{0}(\mathbb{C} \backslash J) \cap \mathcal{C}(\mathbb{C})$ tel que $F \neq 0$. Donc $F$ ne s'étend pas 
analytiquement à $\mathbb{C}$. Soit $f=F_{\mid \bar{\Omega}}$; on a $\bar{\Omega}=\overline{\mathbb{D}}$ et $f \in \mathcal{A}(\Omega) \backslash \mathcal{H}^{\infty}(\mathbb{D})$. De même que plus haut, $\mathcal{H}^{\infty}(\mathbb{D})$ est $w^{*}$-fermé dans $\mathcal{L}^{\infty}(\Omega)=\mathcal{L}^{\infty}(\mathbb{D})$.

(III) Cet exemple est donné dans [Ga, p. 220] comme exemple d'ouvert borné $\Omega$ tel que $\mathcal{R}(\bar{\Omega}) \neq \mathcal{A}(\Omega)$. On va voir qu'en fait $R(\bar{\Omega})$ n'est pas $w^{*}$-dense dans $\mathcal{A}(\Omega)$. On a $\bar{\Omega}=\overline{\mathbb{D}} \backslash \cup_{n \geq 1} \Delta_{n}^{\circ}$, donc

$$
\stackrel{\circ}{\Omega}=\mathbb{D} \backslash \overline{\cup_{n \geq 0} \Delta_{n}^{\circ}}=\mathbb{D} \backslash\left(J \cup\left(\cup_{n \geq 1} \Delta_{n}\right)\right)=\Omega .
$$

Notons $\lambda_{n}$ le centre du disque $\Delta_{n}$. Pour chaque $n \geq 1$, considérons un cercle $C\left(\lambda_{n}, \rho_{n}\right)$ inclus dans $\Omega\left(\rho_{n}>r_{n}\right)$ tel que $\bar{D}\left(\lambda_{n}, \rho_{n}\right) \cap \bar{D}\left(\lambda_{m}, \rho_{m}\right)=$ $\emptyset$ pour $m \neq n, \bar{D}\left(\lambda_{n}, \rho_{n}\right) \cap J=\emptyset, \sup _{n \geq 1}\left(\left|\lambda_{n}\right|+\rho_{n}\right)<1$ et $\sum_{n=1}^{\infty} \rho_{n}<\infty$. Prenons $r \in] \sup _{n \geq 1}\left(\left|\lambda_{n}\right|+\rho_{n}\right), 1[$ et soit

$$
\nu=d z_{\mid r \mathbb{T}}-\sum_{n=1}^{\infty} d z_{\mid C\left(\lambda_{n}, \rho_{n}\right)} .
$$

Alors $\|\nu\|=2 \pi\left(r+\sum_{n=1}^{\infty} \rho_{n}\right)<\infty$ et $\nu \in \mathcal{M}(\Omega)$. Pour tout $z \in \mathbb{C} \backslash \bar{\Omega}=$ $(\mathbb{C} \backslash \overline{\mathbb{D}}) \cup\left(\cup_{n \geq 1} \Delta_{n}^{\circ}\right)$ on a

$$
\iint_{\Omega} \frac{d \nu(\xi)}{\xi-z}=\int_{r \mathbb{T}} \frac{d \xi}{\xi-z}-\sum_{n \geq 1} \int_{C\left(\lambda_{n}, \rho_{n}\right)} \frac{d \xi}{\xi-z}=0,
$$

donc $\mathcal{C}^{-}(\nu) \equiv 0$ et $\nu \perp \mathcal{R}(\bar{\Omega})$. Comme $\alpha(J) \neq 0$, il existe $F \in \mathcal{H}_{0}(\mathbb{C} \backslash$ $J) \cap \mathcal{C}(\mathbb{C})$ tel que $F^{\prime}(\infty):=\lim _{z \rightarrow \infty} z F(z) \neq 0$. Soit $f=F_{\mid \bar{\Omega}}$; alors $f \in \mathcal{A}(\Omega)$ et

$$
\iint_{\Omega} f(\xi) d \nu(\xi)=\int_{r \mathbb{T}} f(\xi) d \xi=2 i \pi F^{\prime}(\infty) \neq 0 .
$$

Donc il existe $f \in \mathcal{A}(\Omega), \nu \in \mathcal{M}(\Omega)$ tels que $\nu \perp R(\bar{\Omega})$ et $\iint_{\Omega} f d \nu \neq 0$. Soient maintenant $z \in \Omega$ et $\rho>0$ tel que $\bar{B}(z, \rho) \subset \Omega$; on définit $\varphi_{z} \in \mathcal{L}^{1}(\Omega)$ par

$$
\varphi_{z}(\xi)=\frac{1}{\pi \rho^{2}} \chi_{\bar{B}(z, \rho)}(\xi) \quad(\xi \in \Omega)
$$

Si $h \in \mathcal{H}^{\infty}(\Omega)$, on a $\iint_{\Omega} h(\xi) \varphi_{z}(\xi) d m(\xi)=h(z)$. L'application $z \rightarrow \varphi_{z}$ est une application continue de $\Omega$ dans $\mathcal{L}^{1}(\Omega)$. Posons $\varphi=\iint_{\Omega} \varphi_{\xi} d \nu(\xi)$. Pour $h \in \mathcal{H}^{\infty}(\Omega)$, on a

$$
\iint_{\Omega} \varphi(\xi) h(\xi) d m(\xi)=\langle\varphi, h\rangle=\iint_{\Omega}\left\langle\varphi_{\xi}, h\right\rangle d \nu(\xi)=\iint_{\Omega} h(\xi) d \nu(\xi) .
$$

Comme $\varphi \in \mathcal{L}^{1}(\Omega)$ on voit que $\mathcal{R}(\bar{\Omega})$ n'est pas $w^{*}$-dense dans $\mathcal{A}(\Omega)$. 
Remarquons par contre que si $\Omega=\mathbb{D} \backslash \cup_{j=1}^{\infty} D_{j}$ où $\left(D_{j}\right)_{j \geq 1}$ est une suite de disques ouverts de $\mathbb{D}$ tels que $\overline{D_{i}} \cap \overline{D_{j}}=\emptyset$ si $i \neq j$ et tels que tous les points d'accumulation de $\left(D_{j}\right)_{j \geq 1}$ sauf un nombre fini soient dans $\mathbb{T}$, alors $R(\bar{\Omega})$ est $w^{*}$-dense dans $\mathcal{H}^{\infty}(\Omega)$ (cf. [Ga, p. 228]).

Notons $\mathcal{M}_{c}(\Omega)$ l'ensemble des mesures $\nu \in \mathcal{M}(\Omega)$ à support dans $\Omega$.

Proposition 5.4. Soit $\Omega$ un ouvert borné de $\mathbb{C}$. Les deux conditions suivantes sont équivalentes:

(I) $\mathcal{M}_{c}(\Omega) \cap \operatorname{Ker} \mathcal{C}^{-}$est dense dans $\left(\operatorname{Ker} \mathcal{C}^{-},\|\cdot\|\right)$.

(II) $\mathcal{M}_{c}(\Omega, \bar{\partial})$ est $\left(\|\cdot\|_{1},\|\cdot\|\right)$-dense dans $\operatorname{Ker} \mathcal{T}$.

Démonstration: Supposons que (2) soit vérifié et soit $\nu \in \operatorname{Ker} \mathcal{C}^{-}, f=$ $\mathcal{C}^{+}(\nu)$. Alors $\mathcal{T}(f)=0$ et il existe une suite $\left(f_{n}\right)_{n \geq 1}$ d'éléments de $\mathcal{M}_{c}(\Omega, \bar{\partial})$ telle que $\lim _{n \rightarrow \infty}\left\|\bar{\partial} f_{n}-\nu\right\|=0$. On a $\mathcal{C}^{-}\left(\bar{\partial} f_{n}\right)=0$, donc $\bar{\partial} f_{n} \in \operatorname{Ker}^{-} \cap \mathcal{M}_{c}(\Omega)$. Réciproquement si (1) est vérifié, soit $f \in$ $\operatorname{Ker} \mathcal{T}$ et soit $\left(\nu_{n}\right)_{n \geq 1}$ une suite d'éléments de $\operatorname{Ker} \mathcal{C}^{-} \cap \mathcal{M}_{c}(\Omega)$ telle que $\lim _{n \rightarrow \infty}\left\|\nu_{n}-\bar{\partial} f\right\|=0$. Posons $f_{n}=\mathcal{C}^{+}\left(\nu_{n}\right)$. Alors $f_{n}$ est nulle sur la composante connexe non bornée de $\mathbb{C} \backslash \overline{\operatorname{Supp} \nu_{n}}$ donc $f_{n} \in \mathcal{M}_{c}(\Omega, \bar{\partial})$, et $\lim _{n \rightarrow \infty}\left\|f-f_{n}\right\|_{1}=0$.

Théorème 5.5. Si $\Omega$ est un domaine de Jordan borné, alors $\mathcal{M}_{c}(\Omega, \bar{\partial})$ est $\left(\|\cdot\|_{1},\|\cdot\|\right)$-dense dans $\operatorname{Ker} \mathcal{T}$.

Démonstration: Il suffit de démontrer l'assertion (I) de la proposition précèdente. Montrons d'abord qu'elle se ramène au cas du disque unité par une application conforme $f: \mathbb{D} \rightarrow \Omega$. Soit $\nu \in \mathcal{M}(\Omega)$ tel que $\mathcal{C}^{-}(\nu) \equiv$ 0 ; posons $f^{-1}(\nu)(B)=\nu(f(B))$ pour $B$ borélien de $\mathbb{D}$. Il est clair que $f^{-1}(\nu) \in \mathcal{M}(\mathbb{D}), \operatorname{Supp} f^{-1}(\nu)=f^{-1}(\operatorname{Supp} \nu),\left\|f^{-1}(\nu)\right\|=\|\nu\|$ et

$$
\mathcal{C}^{-}\left(f^{-1}(\nu)\right)(z)=\frac{1}{\pi} \iint_{\mathbb{D}} \frac{d f^{-1}(\nu)(\xi)}{z-\xi}=\frac{1}{\pi} \iint_{\Omega} \frac{d \nu(\xi)}{z-f^{-1}(\xi)} \quad(z \notin \overline{\mathbb{D}}) .
$$

D'après le théorème de Carathéodory, comme $\partial \Omega$ est une courbe de Jordan, $f^{-1} \in \mathcal{A}(\bar{\Omega})$. Donc, pour $z \notin \overline{\mathbb{D}}$, l'application $\xi \rightarrow \frac{1}{z-f^{-1}(\xi)}$ appartient à $\mathcal{A}(\bar{\Omega})$. Or, d'après le théorème de Mergelyan, $\mathcal{A}(\bar{\Omega})=\mathcal{R}(\bar{\Omega})$ (et même $\mathcal{P}(\bar{\Omega})$, l'adhérence uniforme des polynômes dans $\mathcal{C}(\bar{\Omega})$ ), et par hypothèse, $\mathcal{C}^{-}(\nu) \equiv 0$, i.e. $\nu \perp \mathcal{R}(\bar{\Omega})$. Donc $\mathcal{C}^{-}\left(f^{-1}(\nu)\right) \equiv 0$. Si (I) est vrai pour le disque unité, il existe une suite $\left(\mu_{n}\right), \mu_{n} \in \mathcal{M}_{c}(\mathbb{D})$, telle que $\mathcal{C}^{-}\left(\mu_{n}\right) \equiv 0$ et $\left\|\mu_{n}-f^{-1}(\nu)\right\| \rightarrow 0$. Posons alors $\nu_{n}(B):=f\left(\mu_{n}\right)(B)=$ $\mu_{n}\left(f^{-1}(B)\right)$ pour $B$ borélien de $\Omega$; de même que plus haut, $\nu_{n} \in \mathcal{M}_{c}(\Omega)$, $\mathcal{C}^{-}\left(\nu_{n}\right) \equiv 0$ et $\left\|\nu_{n}-\nu\right\|=\left\|\mu_{n}-f^{-1}(\nu)\right\| \rightarrow 0$. Donc l'assertion (I) se ramène au cas du disque. 
Soient $\mathcal{H}^{1}(\mathbb{T})$ et $\mathcal{H}^{\infty}(\mathbb{T})$ les espaces de Hardy usuels sur le cercle unité, et notons $\langle f, g\rangle:=\frac{1}{2 i \pi} \int_{\mathbb{T}} f(\xi) g(\xi) d \xi$. Par ce crochet, $\mathcal{L}^{\infty}(\mathbb{T})$ s'identifie au dual de $\mathcal{L}^{1}(\mathbb{T})$, et $\mathcal{H}^{1}(\mathbb{T})^{\perp}=\mathcal{H}^{\infty}(\mathbb{T})$. En particulier, $\left(\mathcal{L}^{1} / \mathcal{H}^{1}\right)^{*}=\mathcal{H}^{\infty}$ pour le crochet $\langle[f], g\rangle:=\langle f, g\rangle,[f]$ désignant la classe de $f \in \mathcal{L}^{1}(\mathbb{T})$ dans $\mathcal{L}^{1} / \mathcal{H}^{1}$. Pour $g \in \mathcal{H}^{\infty}(\mathbb{T})$, notons

$$
\gamma(g)(z)=\frac{1}{2 i \pi} \int_{\mathbb{T}} \frac{g(\xi)}{\xi-z} d \xi \quad(z \in \mathbb{D}) .
$$

Alors, l'opérateur $\gamma: \mathcal{H}^{\infty}(\mathbb{T}) \rightarrow \mathcal{H}^{\infty}(\mathbb{D})$ préserve la norme et $\gamma\left(\mathcal{R}_{z \mid \mathbb{T}}\right)=$ $\mathcal{R}_{z}(z \notin \overline{\mathbb{D}})$. Comme $\mathcal{R}(\overline{\mathbb{D}})=\operatorname{Span}\left\{R_{z}: z \notin \overline{\mathbb{D}}\right\}$ est $w^{*}$ séquentiellement dense dans $\mathcal{H}^{\infty}(\mathbb{D})$, par un petit calcul, $\mathcal{R}(\overline{\mathbb{D}})_{\mid \mathbb{T}}$ est $w^{*}$ séquentiellement dense dans $\mathcal{H}^{\infty}(\mathbb{T})$.

Posons maintenant

$$
T(\nu)=\iint_{\mathbb{D}}\left[R_{z}\right] d \nu(z) \quad(\nu \in \mathcal{M}(\mathbb{D})) .
$$

Il est clair que l'application $z \rightarrow\left[R_{z}\right], \mathbb{D} \rightarrow \mathcal{L}^{1} / \mathcal{H}^{1}$ est continue, et que $\left\|\left[R_{z}\right]\right\| \leq 1$; de sorte que l'opérateur $T: \mathcal{M}(\mathbb{D}) \rightarrow \mathcal{L}^{1} / \mathcal{H}^{1}$ est une contraction. En outre, il est immédiat que

$$
\langle T(\nu), g\rangle=\iint_{\mathbb{D}} \gamma(g)(z) d \nu(z) \quad\left(g \in \mathcal{H}^{\infty}(\mathbb{T}), \nu \in \mathcal{M}(\mathbb{D})\right),
$$

et

$$
\left\langle T(\nu), R_{\xi}\right\rangle=-\pi \mathcal{C}(\nu)(\xi) \quad(\xi \notin \overline{\mathbb{D}}, \nu \in \mathcal{M}(\mathbb{D})) .
$$

En particulier,

$$
T(\nu) \equiv 0 \Longleftrightarrow \mathcal{C}^{-}(\nu) \equiv 0 \quad(\nu \in \mathcal{M}(\mathbb{D})) .
$$

Si en outre $\nu \in \mathcal{M}_{c}(\mathbb{D})$, par la formule de Cauchy,

$$
\mathcal{C}(\nu)(\xi)=-\frac{1}{2 i \pi} \int_{\mathbb{T}} \frac{\mathcal{C}(\nu)(z)}{z-\xi} d z=-\left\langle\left[\mathcal{C}(\nu)_{\mid \mathbb{T}}\right], R_{\xi}\right\rangle \quad(\xi \notin \overline{\mathbb{D}}),
$$

de sorte que

$$
T(\nu)=\pi\left[\mathcal{C}(\nu)_{\mid \mathbb{T}}\right] \quad\left(\nu \in \mathcal{M}_{c}(\mathbb{D})\right) .
$$

Soit maintenant $\nu \in \operatorname{Ker} \mathcal{C}^{-}$; et pour $0<r<1$, posons $\nu_{r}=\nu_{\mid r \mathbb{D}}$. On suppose que $\left\|\nu_{r}-\nu\right\| \neq 0$, car sinon $\nu \in \mathcal{M}_{c}(\mathbb{D})$. Comme $\left\|T\left(\nu_{r}\right)\right\|=$ $\left\|T\left(\nu_{r}\right)-T(\nu)\right\| \leq\left\|\nu_{r}-\nu\right\|$, il existe $h \in \mathcal{H}^{1}(\mathbb{T})$ tel que $\left\|\mathcal{C}\left(\nu_{r}\right)+h\right\|_{\mathcal{L}^{1}(\mathbb{T})}<$ $\frac{2}{\pi}\left\|\nu_{r}-\nu\right\|$. En fait, il existe $r<\rho<1$ tel que

$$
\left\|\mathcal{C}\left(\nu_{r}\right)+h\right\|_{\mathcal{L}^{1}(\rho \mathbb{T})}<\frac{2}{\pi}\left\|\nu_{r}-\nu\right\|,
$$


car

$$
\begin{aligned}
\left\|\mathcal{C}\left(\nu_{r}\right)+h\right\|_{\mathcal{L}^{1}(\rho \mathbb{T})} & =\rho \int_{\mathbb{T}}\left|\mathcal{C}\left(\nu_{r}\right)(\rho \xi)+h(\rho \xi)\right||d \xi| \\
\leq & \rho \int_{\mathbb{T}}\left|\mathcal{C}\left(\nu_{r}\right)(\rho \xi)-\mathcal{C}\left(\nu_{r}\right)(\xi)\right||d \xi| \\
& +\rho \int_{\mathbb{T}}\left|\mathcal{C}\left(\nu_{r}\right)(\xi)+h(\xi)\right||d \xi| \\
& +\rho \int_{\mathbb{T}}|h(\rho \xi)-h(\xi)||d \xi| .
\end{aligned}
$$

La première et la troisième intégrale convergeant vers 0 , et la deuxième vers $\left\|\mathcal{C}\left(\nu_{r}\right)+h\right\|_{\mathcal{L}^{1}(\mathbb{T})}$, on a bien

$$
\limsup _{\rho \rightarrow 1^{-}, r<\rho<1}\left\|\mathcal{C}\left(\nu_{r}\right)+h\right\|_{\mathcal{L}^{1}(\rho \mathbb{T})} \leq\left\|\mathcal{C}\left(\nu_{r}\right)+h\right\|_{\mathcal{L}^{1}(\mathbb{T})} .
$$

Posons

$$
d \mu_{r}=d \nu_{r}-\left\{\frac{1}{2 i} \mathcal{C}\left(\nu_{r}\right)_{\mid \rho \mathbb{T}}+\frac{1}{2 i} h_{\mid \rho \mathbb{T}}\right\} d \xi ;
$$

alors $\mu_{r} \in \mathcal{M}_{c}(\mathbb{D})$,

$$
\begin{gathered}
\mathcal{C}^{-}\left(\left\{\frac{1}{2 i} \mathcal{C}\left(\nu_{r}\right)_{\mid \rho \mathbb{T}}\right\} d \xi\right)(z)=\frac{1}{2 i \pi} \int_{\rho \mathbb{T}} \frac{\mathcal{C}\left(\nu_{r}\right)(\xi)}{z-\xi} d \xi=\mathcal{C}^{-}\left(\nu_{r}\right)(z), \\
\mathcal{C}^{-}\left(\left\{\frac{1}{2 i} h_{\mid \rho \mathbb{T}}\right\} d \xi\right)(z)=\frac{1}{2 i \pi} \int_{\rho \mathbb{T}} \frac{h(\xi)}{z-\xi} d \xi=0,
\end{gathered}
$$

et $\left\|\mu_{r}-\nu_{r}\right\|=\frac{1}{2}\left\|\mathcal{C}\left(\nu_{r}\right)+h\right\|_{\mathcal{L}^{1}(\rho \mathbb{T})} \leq \frac{1}{\pi}\left\|\nu_{r}-\nu\right\| \rightarrow 0$. Donc $\mu_{r} \in \mathcal{M}_{c}(\mathbb{D})$, $\mathcal{C}^{-}\left(\mu_{r}\right) \equiv 0$ et $\left\|\mu_{r}-\nu\right\| \rightarrow 0$; ce qu'il fallait démontrer.

Remarque 5.6. Ce théorème s'étend aisément aux domaines bornés dont le bord est une réunion finie disjointe de courbes de Jordan. Voir [R2] pour la théorie des espaces de Hilbert sur les domaines finiment connexes.

Corollaire 5.7. Soit $\Omega$ un domaine de Jordan; alors $\mathcal{D}(\Omega)$ est $\left(v_{c}, v\right)$-séquentiellement dense dans $\operatorname{Ker} \mathcal{T}$.

Démonstration: D'après la Proposition 5.1, l'adhérence séquentielle de $\mathcal{D}(\Omega)$ pour la topologie $\left(\|\cdot\|_{1}, v\right)$ est contenue dans $\operatorname{Ker} \mathcal{T}$. On va montrer l'inclusion réciproque. Soit $f \in \operatorname{Ker} \mathcal{T}$; d'après le Théorème 5.5, il existe une suite $\left(f_{n}\right)_{n \geq 1}$ de $\mathcal{M}_{c}(\Omega, \bar{\partial})$ telle que

$$
\lim _{n \rightarrow \infty}\left\|f_{n}-f\right\|_{1}+\left\|\bar{\partial} f_{n}-\bar{\partial} f\right\|=0 .
$$


Comme $\mathcal{C}_{0}(\Omega)$ est séparable, la topologie $v$ est métrisable sur les parties bornées de $\mathcal{M}(\Omega)$. On pose

$$
\mathcal{M}(\Omega, \bar{\partial})=\left\{g \in \mathcal{L}^{1}(\Omega): \bar{\partial} g \in \mathcal{M}(\Omega)\right\} .
$$

Soient $c>0$, et

$$
B=\left\{g \in \mathcal{M}(\Omega, \bar{\partial}):\|g\|_{1} \leq\|f\|_{1}+c,\|\bar{\partial} g\| \leq\|\bar{\partial} f\|+c\right\},
$$

et soit $d$ une distance sur $B$ définissant la topologie $\left(\|\cdot\|_{1}, v\right)$ sur $B$. Pour $n$ assez grand, $f_{n} \in B$ et $\lim _{n \rightarrow \infty} d\left(f_{n}, f\right)=0$. Pour $s>0$, soit $\theta_{s} \in \mathcal{C}^{\infty}(\mathbb{C})$ telle que Supp $\theta_{s} \subset s \overline{\mathbb{D}}$,

$$
\iint_{\mathbb{C}} \theta_{s}(\xi) d m(\xi)=1 \quad \text { et } \quad 0 \leq \theta_{s}(\xi) \leq 1 \quad(\xi \in \mathbb{C}) .
$$

Fixons $n$ tel que $f_{n} \in B$, et soit $s>0$ assez petit tel que $\operatorname{Supp} \theta_{s} * f_{n} \subset \Omega$. On a $\theta_{s} * f_{n} \in \mathcal{C}^{\infty}(\Omega)$. Comme $\left\|\theta_{s} * f_{n}\right\|_{1} \leq\left\|f_{n}\right\|_{1} \leq\|f\|_{1}+c$ et $\left\|\bar{\partial}\left(\theta_{s} * f_{n}\right)\right\|=\left\|\theta_{s} * \bar{\partial} f_{n}\right\| \leq\|\bar{\partial} f\|+c$, il vient $\theta_{s} * f_{n} \in B$. On a $\lim _{n \rightarrow \infty}\left\|\theta_{s} * f_{n}-f_{n}\right\|_{1}=0$, et il est bien connu que, pour $\mu \in \mathcal{M}(\mathbb{C})$, on a $\lim _{s \rightarrow 0^{+}} \theta_{s} * \mu=\mu$ pour la topologie de la convergence simple sur $\mathcal{C}_{C}(\mathbb{C})$ (ou topologie vague). Donc $\lim _{s \rightarrow 0^{+}} d\left(\theta_{s} * f_{n}, f_{n}\right)=0$. Pour $k \geq 1$, on peut donc construire $n_{k} \in \mathbb{N}$ tel que $f_{n_{k}} \in B$, et $s_{k}>0$ tel que

$$
\theta_{s_{k}} * f_{n_{k}} \in \mathcal{C}_{c}^{\infty}(\Omega) \cap B \quad \text { et } \quad d\left(\theta_{s_{k}} * f_{n_{k}}, f\right)<\frac{1}{k} .
$$

Donc Ker $\mathcal{T}$ coïncide avec l'adhérence séquentielle de $\mathcal{D}(\Omega)$ dans $\left(\mathcal{M}(\Omega, \bar{\partial}),\left(\|\cdot\|_{1}, v\right)\right)$. A fortiori, $\operatorname{Ker} \mathcal{T}$ coïncide avec l'adhérence séquentielle de $\mathcal{D}(\Omega)$ dans $\left(\mathcal{M}_{\text {loc }}(\Omega, \bar{\partial}),\left(v_{c}, v\right)\right)$.

Remarque 5.8. Soit $\Omega$ un ouvert borné de $\mathbb{C}$ tel que les composantes connexes de son complémentaire soient d'intérieur non vide. Notons $\mathcal{C}^{\infty}(\bar{\Omega})$ l'ensemble des restrictions à $\Omega$ des fonctions $\mathcal{C}^{\infty}$ au voisinage de $\bar{\Omega}$ (ce qui correspond à la définition habituelle quand $\partial \Omega$ est une sous-variété de classe $\mathcal{C}^{\infty}$ de $\left.\mathbb{C}\right)$; alors $\mathcal{C}^{\infty}(\bar{\Omega})$ est séquentiellement $\left(v_{c}, v_{\infty}\right)$-dense dans $\mathcal{M}_{\text {loc }}(\Omega, \bar{\partial})$. En effet, soit $f \in \mathcal{M}_{\text {loc }}(\Omega, \bar{\partial})$; d'après le théorème de Runge, il existe une suite $\left(R_{n}\right)_{n \geq 0}$ de fonctions rationnelles à pôles hors de $\bar{\Omega}$ tel que $\lim _{n \rightarrow \infty} R_{n}=f-\overline{\mathcal{C}}^{+}(\bar{\partial} f)$ dans $\mathcal{L}_{\text {loc }}^{1}(\Omega)$. Soit $\left(s_{n}\right)_{n \geq 0}$ une suite de réels positifs tendant vers 0 , et soit

$$
f_{n}=R_{n}+\mathcal{C}\left(\theta_{s_{n}} * \bar{\partial} f\right)_{\mid \Omega} .
$$


On a $f_{n} \in \mathcal{C}^{\infty}(\bar{\Omega})$. Comme $\mathcal{C}\left(\bar{\partial} f * \theta_{s_{n}}\right)=\mathcal{C}(\bar{\partial} f) * \theta_{s_{n}} \underset{n \rightarrow \infty}{\longrightarrow} \mathcal{C}(\bar{\partial} f)$ dans $\mathcal{L}_{\text {loc }}^{1}(\mathbb{C})$, on a $\left\|\mathcal{C}\left(\bar{\partial} f * \theta_{s_{n}}\right)_{\mid \Omega}-\mathcal{C}^{+}(\bar{\partial} f)\right\|_{1} \underset{n \rightarrow \infty}{\longrightarrow} 0$, et

$$
\begin{aligned}
\lim _{n \rightarrow \infty}\left(f_{n}-f\right)=\lim _{n \rightarrow \infty}\left(R_{n}-f\right. & \left.+\mathcal{C}^{+}(\bar{\partial} f)\right) \\
& +\lim _{n \rightarrow \infty}\left(\mathcal{C}\left(\theta_{s_{n}} * \bar{\partial} f\right)_{\mid \Omega}-\mathcal{C}^{+}(\bar{\partial} f)\right)=0
\end{aligned}
$$

dans $\mathcal{L}_{\text {loc }}^{1}(\Omega)$, et à fortiori dans $\left(\mathcal{L}_{\text {loc }}^{1}(\Omega), v_{c}\right)$. D'autre part,

$$
\bar{\partial}\left(f_{n}-f\right)=\left(\theta_{s_{n}} * \bar{\partial} f\right)_{\mid \Omega}-\bar{\partial} f .
$$

Soit $\varphi \in \mathcal{C}_{b}(\Omega)$; d'après le théorème de Fubini,

were

$$
\int_{\Omega} \varphi(\xi)\left(\theta_{s_{n}} * \bar{\partial} f\right)(\xi) d m(\xi)=\int_{\Omega} \psi_{s_{n}}(\eta) d(\bar{\partial} f)(\eta),
$$

$$
\psi_{s_{n}}(\eta)=\int_{\Omega} \theta_{s_{n}}(\xi-\eta) \varphi(\xi) d m(\xi) .
$$

Comme $\lim _{n \rightarrow \infty} \psi_{s_{n}}(\eta)=\varphi(\eta)$ pour $\eta \in \Omega$, d'après le théorème de convergence dominée,

$$
\lim _{n \rightarrow \infty} \int_{\Omega} \psi_{s_{n}}(\eta) d(\bar{\partial} f)(\eta)=\int_{\Omega} \varphi(\xi) d(\bar{\partial} f)(\xi) .
$$

Donc $\lim _{n \rightarrow \infty} \bar{\partial} f_{n}=\bar{\partial} f$ dans $\left(\mathcal{M}(\Omega), v_{\infty}\right)$ et $\lim _{n \rightarrow \infty} f_{n}=f$ dans $\left(\mathcal{M}_{\text {loc }}(\Omega, \bar{\partial}),\left(v_{c}, v_{\infty}\right)\right)$.

Dans le cas où $\Omega$ est un domaine de Jordan, on voit alors que $\mathcal{T}$ est l'extension par continuité à $\left(\mathcal{M}_{\text {loc }}(\Omega, \bar{\partial}),\left(v_{c}, v\right)\right)$ de l'application $f \rightarrow$ $f_{\mid \partial \Omega} \operatorname{sur} \mathcal{C}^{\infty}(\bar{\Omega})$, et $\operatorname{Ker} \mathcal{T}$ est l'adhérence séquentielle dans $\left(\mathcal{M}_{\text {loc }}(\Omega, \bar{\partial})\right.$, $\left.\left(v_{c}, v\right)\right)$ de $\mathcal{D}(\Omega)$. On a donc une trace au sens le plus classique du terme (cf. [LM, Chapitre 1], [Gr], et $[\mathbf{V}]$ ).

\section{Références}

[BG1] C. A. Berenstein et R. Gay, "Complex variables. An introduction", Graduate Texts in Mathematics 125, Springer-Verlag, New York, 1991.

[BG2] C. A. Berenstein et R. Gay, "Complex analysis and special topics in harmonic analysis", Springer-Verlag, New York, 1995.

[B] H. Brezis, "Analyse fonctionnelle", Théorie et applications, Collection Mathématiques Appliquées pour la Maîtrise, Masson, Paris, 1983.

[C] J. B. Conway, "Functions of one complex variable. II", Graduate Texts in Mathematics 159, Springer-Verlag, New York, 1995. 
[D] G. DAvid, Opérateurs intégraux singuliers sur certaines courbes du plan complexe, Ann. Sci. École Norm. Sup. (4) 17(1) (1984), $157-189$.

[EV1] J. Esterle et A. Volberg, Sous-espaces invariants par translations bilatérales de certains espaces de Hilbert de suites quasianalytiquement pondérées, C. R. Acad. Sci. Paris Sér. I Math. 326(3) (1998), 295-300.

[EV2] J. Esterle et A. Volberg, Asymptotically holomorphic functions and translation invariant subspaces of weighted Hilbert spaces of sequences, Ann. Sci. École Norm. Sup. (4) 35(2) (2002), 185-230.

[Ga] T. W. Gamelin, "Uniform algebras", Prentice-Hall, Inc., Englewood Cliffs, N. J., 1969.

[Go] G. M. Goluzin, "Geometric theory of functions of a complex variable", Translations of Mathematical Monographs 26, American Mathematical Society, Providence, R.I., 1969.

[Gr] P. GRIsvard, "Elliptic problems in nonsmooth domains", Monographs and Studies in Mathematics 24, Pitman (Advanced Publishing Program), Boston, MA, 1985.

[G] A. Grothendieck, Sur certains espaces de fonctions holomorphes. I, J. Reine Angew. Math. 192 (1953), 35-64.

[H] I. Harlouchet, Traces de Cauchy, fonctions asymptotiquement holomorphes et algèbres de Beurling quasyanalytiques, Thèse de doctorat, Université Bordeaux I (1999).

[LM] J.-L. Lions ET E. Magenes, "Problèmes aux limites non homogènes et applications", Vol. 1, Travaux et Recherches Mathématiques 17, Dunod, Paris, 1968.

[Na] I. P. NAtANSON, "Theory of functions of a real variable", Vol. II, Translated from the Russian by Leo F. Boron, Frederick Ungar Publishing Co., New York, 1961.

[N] N. K. NIKOL'SKII , Problèmes d'analyse et synthèse spectrale, Cours Post-DEA, Bordeaux (1993).

[P] I. I. PRIVALOv, "Graničnye svořstva analitičeskih funkciı", (Russian) [Boundary properties of analytic functions, 2d ed.] Gosudarstv. Izdat. Tehn.-Teor. Lit., Moscow-Leningrad, 1950.

[Ra] Th. RAnsford, "Potential theory in the complex plane", London Mathematical Society Student Texts 28, Cambridge University Press, Cambridge, 1995.

[R] W. Rudin, "Real and complex analysis", Third edition, McGrawHill Book Co., New York, 1987. 
[R2] W. Rudin, Analytic functions of class $H_{p}$, in: "Lectures on functions of a complex variable", The University of Michigan Press, Ann Arbor, 1955, pp. 387-397.

[Sa] T. S. SAlimov, The $A$-integral and boundary values of analytic functions, (Russian), Mat. Sb. (N.S.) 136(178) (1988), no. 1, 24-40, 160; translation in: Math. USSR-Sb. 64(1) (1989), 23-39.

[S] R. V. Sibilev, A uniqueness theorem for Wolff-Denjoy series, (Russian. Russian summary), Algebra i Analiz 7(1) (1995), 170-199; translation in: St. Petersburg Math. J. 7(1) (1996), $145-168$.

[Tr] C. TRICOT, "Curves and fractal dimension", With a foreword by Michel Mendès France, Translated from the 1993 French original, Springer-Verlag, New York, 1995.

[T] M. TsusI, "Potential theory in modern function theory", Reprinting of the 1959 original, Chelsea Publishing Co., New York, 1975.

[U] P. L. UL'JAnov, Integrals of Cauchy type, (Russian), Trudy Mat. Inst. Steklov. 60 (1961), 262-281.

[V] K. Vo-Khac, "Distributions. Analyse de Fourier. Opérateurs aux dérivées partielles", Tomes 1 et 2, Librairie Vuibert, 1972.

Department of Mathematics

NTNU

7491 Trondheim

Norway

E-mail address: iban_hm@yahoo.fr

Primera versió rebuda el 10 de desembre de 2002, darrera versió rebuda el 5 de març de 2003. 